\title{
Using viscoelastic properties to quantitatively estimate the amount of modified poly(lactic acid) chains through reactive extrusion
}

\author{
Jonathan Cailloux, ${ }^{\text {a) }}$ Orlando O. Santana, ${ }^{\text {b) }}$ and Maria Ll. Maspoch ${ }^{\text {c) }}$ \\ Centre Català del Plàstic (CCP) - Universitat Politècnica de Catalunya \\ (ETSEIB-UPC), C/ Colom, 114, Terrassa 08222, Spain
}

Jordi J. Bou ${ }^{\text {d) }}$

Department of Chemical Engineering - Universitat Politècnica de Catalunya (ETSEIB-UPC), Pavelló G, planta 1, Avenida Diagonal, 647, Barcelona 08028, Spain

\author{
Felix Carrasco ${ }^{\mathrm{e}}$ \\ Department of Chemical Engineering - Universitat de Girona (UdG), \\ Campus Montilivi s/n, Girona 17071, Spain
}

(Received 3 January 2015; final revision received 16 June 2015; published 12 August 2015)

\begin{abstract}
In this study, the dynamic viscoelastic properties of three structurally modified poly(lactic acid) (PLA) samples processed through reactive extrusion (REX) were analyzed. While classical chromatographic and spectroscopic techniques exhibited limited sensitivity to the presence of topological changes, rheological measurements confirmed the presence of nonuniform branched macromolecules, holding sparsely long chain branches. According to the processing conditions used, the flow activation energy and the thermorheologically simple behavior remained roughly unaffected for PLA-REX containing an amount of modified chains up to $24 \%$. Distinctly separated relaxation processes in a broader transition zone were observed in the complex viscosity function $\left(\left|\eta^{*}(\omega)\right|\right)$ of all PLA-REX samples. The "extended Carreau-Yassuda" model and an extension of the Havriliak-Negami model, proposed in this work, were used to capture the main characteristics of $\left|\eta^{*}(\omega)\right|$ experimental data. Both fitted models were inverted to molecular weight distribution (MWD) spectrum using the numerical inversion technique of Shaw and Tuminello, and these were compared with size exclusion chromatography MWDs. It was shown that the resolution of the predicted bimodal MWDs was enhanced when the model used to fit $\left|\eta^{*}(\omega)\right|$ data was exempted from the Cox-Merz rule and included a complex time dependence.

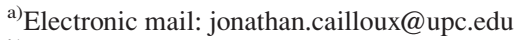

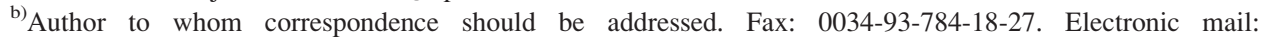
orlando.santana@upc.edu

${ }^{c}$ Electronic mail: maria.lluisa.maspoch@upc.edu

${ }^{\mathrm{d})}$ Electronic mail: jordi.bou@upc.edu

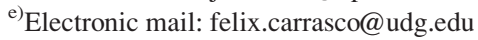


Based on the MWDs deduced from solely melt measurements, a procedure was described to quantitatively estimate the amount of modified chains. (C) 2015 The Society of Rheology.

[http://dx.doi.org/10.1122/1.4928071]

\section{INTRODUCTION}

Since branching significantly affects both the polymer flow properties and its processing behavior, the characterization of the polymer architecture on a molecular level is of significant importance. Gel permeation chromatography (GPC) coupled with light scattering (LS) and intrinsic viscosmetry (IV) yield information on the shape and size of branched macromolecules in carefully prepared polymers with well-defined structures [i.e., low density polyethylenes (LDPE)] [Wang et al. (2004); Gabriel and Munstedt (2002); Gabriel et al. (2002); Wood-Adams et al. (2000)]. In solution, due to a more compact configuration (i.e., coil contraction), the radius of gyration $R_{g}$, which is a measure of the polymer's hydrodynamic volume using LS, is smaller for a long chain branched (LCB) macromolecule than its linear counterpart of similar molecular weight (MW). Thus, the ratio of the radii of gyration of a branched, $R_{g, B}$, to that of a linear polymer, $R_{g, L}$, of similar MW in solution expresses the degree of branching, $g$, as given as [McKee et al. (2005); Wang et al. (2004)]

$$
g=\frac{R_{g, B}^{2}}{R_{g, L}^{2}} .
$$

Similarly, the Zimm-Stockmayer method [Zimm and Stockmayer (1949)] allows for the estimation of the contraction factor $g^{\prime}$. It is defined as the ratio of the intrinsic viscosity, which is a measure of the polymer's hydrodynamic volume using IV, of the branched polymer $[\eta]_{B}$ to that of a linear polymer $[\eta]_{L}$ of equivalent MW, as given as follows [McKee et al. (2005); Wang et al. (2004)]:

$$
g^{\prime}=\frac{[\eta]_{B}^{2}}{[\eta]_{L}^{2}} .
$$

While $g$ and $g^{\prime}$ equal 1 for linear polymers, both parameters decrease with an increase in the degree of LCB. Nevertheless, this procedure fails to characterize sparsely and/or nonuniform branched polymers in MW, in architectures and in the number of branch points per macromolecules [i.e., sparsely LCB high density metallocene catalyzed polyethylene (LCB-mHDPE)]. This is because the experimental sensitivity is insufficient to estimate the slight differences in molecular dimensions between the low amount of nonuniform branched macromolecules and the dominant linear fraction. $g$ and $g^{\prime}$ remain extremely close to 1 within the experimental error, and the estimation of the degree of LCB is not feasible [Janzen and Colby (1999); Kratochvil (2000); Garcia-Franco et al. (2008); Kratochvil (2000); Stadler and Munstedt (2008a)].

Complementary information about the number of branches can be obtained using the high resolution nuclear magnetic resonance (NMR). Unfortunately, this method exhibits a limited sensitivity to sparsely and/or nonuniform branched macromolecules similar to that of the chromatographic method. The number of branch points cannot be counted below the usual detection limit of about 1 branch per $10^{4}$ carbon atoms, as already reported by Janzen and Colby (1999), Shroff and Mavridis (1999), Wood-Adams et al. (2000), and Malmberg et al. (2002) in transition metal-catalyzed HDPE. Moreover, this 
technique cannot distinguish branches of six carbon atoms length and longer, even though this length has been increased to at least ten carbon atoms under specific testing conditions [Liu et al. (1999)]. The source of trouble is the physical reality that NMR cannot differentiate between the magnetic resonance properties of long branches and the polymer backbone because they give rise to the same spectral pattern irrespective of chain length [Vega et al. (1998); Janzen and Colby (1999); Crosby et al. (2002)].

On the other hand, melt rheological properties exhibit a high sensitivity to the presence of LCB, even at concentrations below the detection limits of classical spectroscopic or chromatographic methods. This is due to significant differences between the relaxation behavior of linear and LCB chains. Thus, rheological measurements remain one of the most useful methods to obtain large-scale molecular information. Obtaining molecular information about LCB using only rheology-based methods is not trivial. Numerous studies reported that increasing the MW, broadening the MW distribution (MWD), or increasing the content of LCB have similar effects on the shear viscosity function [Shroff and Mavridis (1999); Vega et al. (2002); Doerpinghaus and Baird (2003); Shroff and Mavridis (1999); van Ruymbeke et al. (2005); Stadler and Munstedt (2008b)]. That is, the distribution of relaxation times is not a unique function of chain architecture. The separation of the MW and polydispersity effects from that of LCB is an important prerequisite in order to correlate branching topology and rheological properties.

On this basis, different quantitative correlations based solely on melt measurements have been utilized to estimate the degree of LCB. Trinkle et al. (2002) and GarciaFranco et al. (2008) used the so-called van Gurp-Palmen (vGP) plot to estimate low levels of LCB in various polydisperse polyolefin systems. Another commonly used method to relate shear rheological properties to LCB level is based on the "Dow rheology index," which was specifically designed for polyolefins with narrow and similar MWDs and hence cannot be used otherwise [Lai et al. (1994)].

In contrast to other rheological properties, which are affected by both the broadening of the MWD and the presence of LCB, the dependence of the zero-shear rate viscosity $\left(\eta_{0}\right)$ on the weight-average MW $\left(M_{w}\right)$ [cf. Eqs. (3a) and (3b)] has been reported as independent of the distribution of MW for linear PE [Munstedt and Auhl (2005); Stadler et al. (2006); Stadler and Munstedt (2008a)], polystyrene [Munstedt (1980)], and polypropylene [Fleissner (1992)]. Consequently, the deviation from Eq. (3), which has been established for linear polymers, is a good indicator of the presence of LCB, as already reported by Janzen and Colby (1999), Wood-Adams et al. (2000), Gabriel et al. (2002), Auhl et al. (2004), Stadler et al. (2006), Piel et al. (2006), and Stadler and Munstedt (2008b)

$$
\begin{aligned}
& \eta_{0}=K_{1} M_{W} \text { for } M_{w}<M_{c}, \\
& \eta_{0}=K_{2} M_{W}^{\alpha} \text { for } M_{w}>M_{c},
\end{aligned}
$$

where $K_{1}$ and $K_{2}$ are parameters dependent on both the polymer species and the temperature. $\alpha$ usually ranges between 3.4 and 3.6 for linear polymers. $M_{c}$ is the critical MW for entanglements.

The estimation of the flow activation energy, $E_{a}$, is another example of LCB characterization by considering rheological measurements alone. The activation energy for viscous flow is affected by chain flexibility, intermolecular interactions, the concentration of polar groups, and side-chain branches. While the presence of LCB yields an increase in both the temperature dependence and $E_{a}$, the thermorheological behavior becomes complex [Malmberg et al. (2002); Lohse et al. (2002); Gotsis et al. (2004)]. That is, the 
sequence of the molecular relaxations is temperature dependent. Unfortunately, the situation is ambiguous because the absence of an enhanced $E_{a}$ and/or the loss of simple thermorheological behavior do not necessarily exclude the presence of low amounts of LCB, as already reported in Wasserman and Graessley (1996), Vega et al. (1998), and Shroff and Mavridis (1999) for sparsely branched HDPE resins and Munari et al. (1990) for branched poly(butyleneisophthalate). Therefore, the flow activation energy data alone cannot always indicate the presence of LCB.

Several authors also attempted to combine shear rheology and spectroscopic or chromatographic methods. Shroff and Mavridis (1999), Janzen and Colby (1999), and Wang et al. (2004) coupled size exclusion chromatography (SEC)-viscosmetry technique with shear rheology to detect LCB. Wood-Adams and Dealy (2000) reported a technique to estimate the level of LCB by comparing MWDs measured by SEC with MWDs inferred from the inversion technique of the viscosity data proposed by Shaw and Tuminello (1994). Although promising results were obtained for detecting branching down to $0.005 \mathrm{LCB}$ per 1000 carbon atoms, final LCB content estimations were based on NMR experiments.

SEC is frequently placed at the forefront of molecular characterization in polymer science. However, this technique is time consuming, expensive (use of solvents) and experimental data may be flawed by coelution issues. Thus, an increasing interest has grown to infer MWD from rheological measurements. This technique provides specific advantages as compared to SEC. For instance, standard rheological property measurements are less time consuming than SEC elution curve analysis. The previous dissolution of the polymer, which can be the rate-limiting step for analysis time, is not necessary to measure viscoelastic properties. Benefiting from convenient experimental procedures and higher resolution with respect to high MW (HMW) components, this technique may be used to perform high throughput process controls in industrial settings. However, it should be kept in mind that this method cannot replace SEC measurements. Both methods have to be used simultaneously in order to complete each other.

The manipulation of rheological raw data to obtain an accurate MWD is not a trivial operation. Among the numerous procedures to deduce MWD from melt measurements [Tuminello (1986); Mead (1994); Wasserman (1995); Dealy and Larson (2006a); Zhang et al. (2007)], the numerical models, which relate the viscosity function data to the MWD, have received much attention. The simpler transformation procedures are based on an assumed bulk mixing rules of linear architectures as given as [Bersted and Slee (1977); Malkin and Teishev (1991)]

$$
\eta^{1 / \alpha}=\sum w_{i} \eta_{i}^{1 / \alpha}
$$

where $\alpha$ is the exponent in the power-law dependence between $\eta_{0}$ and $M_{w}$ (often set to 3.4). $w_{\mathrm{i}}$ is the weight fraction of component $i$ and $\eta_{i}$ its corresponding viscosity. A feature of the above mixing rule is that the coupling effects between individual components with different chain lengths are taken into account.

While there are valid reasons to question the detailed assumptions leading to this mixing rule, Tuminello and Cudremauroux (1991) reported satisfactory results to predict MWDs from complex dynamic viscosity data of polystyrene. To contribute to the determination of MWD from raw viscosity data, Malkin and Teishev (1991) developed an explicit differential solution [(Eq. (5)] based on Eq. (4)

$$
W(m)=1+\left.\frac{1}{\nu}\left(\frac{\eta}{\eta_{0}}\right)^{\frac{1}{\alpha}}\left(\frac{\dot{\gamma}}{\gamma_{c}}\right)^{\frac{\nu}{\alpha}} \frac{d \ln \eta}{d \ln \dot{\gamma}}\right|_{\dot{\gamma}=\dot{\gamma}_{c} m^{-\alpha / \nu}},
$$


where $W(m)$ is the cumulative MWD. That is, the cumulative weight fraction of polymer up to a reduced MW, $m$ [cf. Eq. (6)], which is defined as the ratio of the MW, $M$, to that of $M_{w} .-\nu$ is the slope of the power-law region. $\dot{\gamma}$ is the shear rate and $\dot{\gamma}_{c}$ is the critical shear rate which is a horizontal scaling parameter fixing the position of the transition region between the low shear rate plateau and the power-law regime

$$
m=\frac{M}{M_{w}} .
$$

Nevertheless, authors were unable to maintain important features of the MWD (e.g., bimodality) during calculations. Shaw and Tuminello (1994) showed that this protocol failed due to the loss of important molecular information during viscosity curve fitting. Since bimodality is of major importance for the performance of a polymer, Shaw and Tuminello (1994) developed a numerical method to deduce MWD from shear $(\eta(\dot{\gamma}))$ or complex viscosity $\left(\left|\eta^{*}(\omega)\right|\right)$ data from Eq. (5). As compared to other robust protocols based on the inversion of the dynamic modulus [Nobile and Cocchini (2001); Cocchini and Nobile (2003); Chen and Zhang (2012)], this technique allows the inversion of the melt viscosity data with no a priori assumptions about plateau modulus values or about the shape of the MWD prior to analysis.

While the numerical procedure proposed by Shaw and Tuminello (1994) to infer MWD from viscosity functions was developed for linear flexible polymers, it is believed that this method could be extended to LCB polymers using oscillatory shear rheological measurements. Precise and complete viscosity data over a wide range of frequency are required to avoid noise issues and false extrapolations. To this end, both phenomenological "Carreau-Yassuda" and "Vinogradov fluidity" models have been used [Wood-Adams and Dealy (1996); Liu et al. (1998)]. Within the linear viscoelastic regime (LVE), the assumption of the validity of the Cox-Merz rule [Eq. (7)] allows for an equivalent form of both viscosity models in terms of $\eta(\dot{\gamma})$ to the magnitude of the complex viscosity $\left(\left|\eta^{*}(\omega)\right|\right)$ determined by dynamic experiments

$$
\eta(\dot{\gamma})=\left|\eta^{*}(\omega)\right| \text { for } \quad \dot{\gamma}=\omega .
$$

The viscosity-MWD moments may be equally deduced either from $\dot{\gamma}$ or from the angular frequency $(\omega)$ dependence of the viscosity model considered. However, Eq. (7) is only valid for linear architectures. Significant discrepancies between both viscosities have been observed for LCB polymers, leading to a failure in the Cox-Merz equality for branched architectures, as already reported by Ferri and Lomellini (1999), Lehermeier and Dorgan (2001), Carreau et al. (2010), and Corre et al. (2011). A different analytical viscoelastic model relating quantitatively oscillatory flow properties to molecular structure and exempted from the above empiricism is, therefore, required to describe the dynamic viscosity function of LCB polymers.

A couple of noteworthy features specific to the viscosity function in LCB polymers are the $\eta_{0}$ increase and the enhanced shear-thinning regime while its onset is shifted to lower frequencies [Vega et al. (1998); Dorgan et al. (1999); Gabriel and Munstedt (2002); Lohse et al. (2002); Doerpinghaus and Baird (2003); Auhl et al. (2004); Stadler et al. (2006)]. Stadler and Munstedt (2008a) also observed several distinct curvatures in the high frequency region. An "extension of the Carreau-Yassuda" model (referred to as CY-Ext) was then introduced to capture the main features of the $\left|\eta^{*}(\omega)\right|$ experimental data. However, the main drawback of this model should arise from its basis. Authors based their extended model on the original phenomenological Carreau-Yassuda model 
(referred to as CY) developed for steady-state viscosity functions (i.e., $\eta(\dot{\gamma}))$ even though the Cox-Merz rule does not hold for branched polymers. Errors in $\left|\eta^{*}(\omega)\right|$ fitting analysis are likely to occur and hence lead to inaccurate viscosity-MWD calculation [inversion procedure of Shaw and Tuminello (1994)]. A simpler mathematical model to parameterize $\left|\eta^{*}(\omega)\right|$ that features all the before mentioned characteristics and exempted from the Cox-Merz assumption has not yet been described in the literature to the best of the author's knowledge.

Turning the high sensitivity of rheology into a quantitative technique, to show LCB and to infer MWD, has been useful in the characterization of polyolefins. However, in today's world of awareness of the environmental situation and of "green" chemistry, biopolyesters [e.g., poly(lactic acid) (PLA)] have gained much attention as a replacement for conventional polymers obtained from petrochemical feed stocks. For the purpose of providing specific processing behavior, their rheological properties may be tailored through the modification of the chain architecture. To this end, reactive extrusion (REX) coupled with a multifunctional reactive agent has been reported as a promising and satisfactory method by several authors [Carlson et al. (1998); Di et al. (2005); Raquez et al. (2008); Sungsanit et al. (2010); Liu et al. (2010); Mihai et al. (2010); Corre et al. (2011)]. However, communications which report the molecular characterization and the MW determination of structurally modified PLA from rheological measurements are rare. Dorgan et al. (2005) provided some insight into the molecular dimensions using the packing model for linear PLA featuring different D-lactide contents.

The current study focuses on the development of a quantitative method to detect and estimate extremely low levels of structural modifications in PLA from rheological measurements. The present manuscript is organized as follows: Section II summarizes the Shaw and Tuminello (1994) numerical procedure to infer MWDs from viscosity data of polydisperse linear polymer melts. In Sec. III, authors discuss the limitations of the CYExt model to parameterize $\left|\eta^{*}(\omega)\right|$ functions of LCB polymers and introduce an extended model exempting from the Cox-Merz empiricism. The viscoelastic properties of three architecturally modified PLA resins processed by REX are analyzed in Sec. V. This section also describes a resolution method to predict bimodal MWDs with a polydispersity index (PDI $=M_{w} / M_{n}$ ) of about 2 from $\left|\eta^{*}(\omega)\right|$ data and reports MWD estimations for linear and modified PLA from melt measurements. The paper concludes with a description of a quantitative procedure to determine the amount of modified PLA chains from solely melt measurements using standard equipments and procedures.

\section{INFERRING CONTINUOUS MWD SPECTRA FROM LVE DATA FOR LINEAR FLEXIBLE POLYMERS}

In the present work, the procedure used to relate $\left|\eta^{*}(\omega)\right|$ to the MWD is based on the numerical approach developed by Shaw and Tuminello (1994) and later refined by Wood-Adams and Dealy (1996) and Liu et al. (1998). According to Shaw and Tuminello (1994), the Cox-Merz assumption was assumed as valid to be used in this approach and $\eta(\dot{\gamma})$ was substituted by $\left|\eta^{*}(\omega)\right|$ and $\dot{\gamma}$ by $\omega$.

Similar to the procedure developed by Malkin and Teishev (1991), $\left|\eta^{*}(\omega)\right|$ is related to the MWD using Eq. (4) which allows the derivation of Eq. (5) in order to correlate $W(m)$ to $\left|\eta^{*}(\omega)\right|$. The logarithmic differential MWD $(w(\log m))$ is then calculated by differentiating Eq. (5) with respect to $\log (m)$. Thus, $w \log (m)$ is the differential form of $W(m)$ [cf. Eq. (5)], $w \log (m)=\mathrm{d} W(m) / \mathrm{d} \log (m)$. Since $m$ is related to $M$ using Eq. (6), $w$ $\log (m)=\mathrm{d} W(m) / \mathrm{d} \log (m)=\mathrm{d} W(\mathbf{M}) / \mathrm{d} \log (M)$ 


$$
w(\log m)=\left[\frac{-\ln (10)}{m v^{2}}\right]\left[\frac{\eta^{*}}{\eta_{0}}\right]^{1 / \alpha}\left[\alpha \frac{\mathrm{d}^{2} \ln \eta^{*}}{\mathrm{~d} \ln \omega^{2}}+v \frac{\mathrm{d} \ln \eta^{*}}{\mathrm{~d} \ln \omega}+\left(\frac{\mathrm{d} \ln \eta^{*}}{\mathrm{~d} \ln \omega}\right)^{2}\right] .
$$

In the high frequency limit, $-\nu$ is the slope of the power-law region. For linear polymers, $\alpha$ represents the exponent in the power-law dependence between $\eta_{0}$ and $M_{w}$ [cf. Eq. (3b)]. $m$ is the reduced MW [cf. Eq. (6)], which is related to $\omega$ using

$$
m=\left(\frac{\omega}{\omega_{c}}\right)^{v / \alpha}
$$

Here, $\omega_{c}$ is the critical frequency, which is a horizontal scaling parameter fixing the position of the transition region between the Newtonian and shear-thinning regime. $\omega_{c}$ is determined by a linear fitting passing through the last decade of the natural log of $\left|\eta^{*}\right|$ as a function of the natural $\log$ of $\omega$. The Yintercept is then used in Eq. (10) to calculate $\omega_{c}$ [Wood-Adams and Dealy (1996)]

$$
\omega_{c}=\exp \left[\left(\ln \eta_{0}-\text { Yintercept }\right) /-v\right] .
$$

Shaw and Tuminello (1994) stated that the polymer MWD may only be estimated from high quality and complete rheological data. A proper MWD estimation requires accurate experimental data between both regimes, since they have to be differentiated twice. Independently of the polymer under study, the accessible range of $\omega$ is usually limited due to equipment limitations. It is not always possible to collect sufficient rheological information for this technique to be successful even though the principle of timetemperature superposition (TTS) is used. Independently of the operator's skillfulness, both between- and within-sample experimental errors also lower the precision of the dynamic data sets. A viable solution in order to reduce noise issues and to extend the range of $\omega$ at both ends is to fit the set of available data using well-known viscosity models.

Regarding linear polymers with narrow MWDs, the CY model [Eq. (11)] has showed sufficient flexibility to fit properly the $\eta(\dot{\gamma})$ curves from the terminal to the shear-thinning regime

$$
\eta(\dot{\gamma})=\eta_{0}\left[1+\left(\tau_{0} \dot{\gamma}\right)^{\alpha}\right]^{(\nu-1) / \alpha}
$$

where $\eta_{0}$ is the zero-shear viscosity which is the limiting value at low $\dot{\gamma}$ of the steadyflow viscosity. $\tau_{0}$ is the characteristic viscous relaxation time, fixing the position of the transition region between the Newtonian and shear-thinning regime. $\alpha$ is the width of this transition and $(\nu-1)$ the slope $d \log \eta / d \log \dot{\gamma}$ of the shear-thinning regime at high $\dot{\gamma}$.

Within the LVE, the applicability of the Cox-Merz rule [Eq. (7)] to linear polymers allows to relate $\eta(\dot{\gamma})$ to $\left|\eta^{*}(\omega)\right|$ in Eq. (9) [Lehermeier and Dorgan (2001); Stadler and Munstedt (2008b)]. A complete $w \log (m)$ spectrum is then obtained when the second derivative in Eq. (8) of the viscosity function extrapolated at both ends decreases below the threshold of 0.005 [Dealy and Larson (2006b)].

For LCB polymers, the ability to predict $\eta(\dot{\gamma})$ from $\left|\eta^{*}(\omega)\right|$ is difficult. A failure in the Cox-Merz equality is relevant for branched architectures, as already reported by Ferri and Lomellini (1999), Carreau et al. (2010), and Corre et al. (2011). Consequently, errors in prediction are expected if $\eta(\dot{\gamma})$ is deduced from dynamic measurements and if viscosity models developed for steady-flow curve are used to parameterize $\left|\eta^{*}(\omega)\right|$ data. 


\section{VISCOSITY MODELS FOR LCB POLYMERS: THEORY AND LIMITATIONS}

Stadler and Munstedt (2008a) introduced an extension of the phenomenological Carreau-Yassuda model [Eq. (12)] (referred to as CY-Ext) in order to fit $\left|\eta^{*}(\omega)\right|$ functions of LCB-mHDPEs featuring several pronounced curvatures

$$
\left|\eta^{*}(\omega)\right|=\eta_{0} \prod_{i=1}^{m}\left(1+\left(\tau_{i} \omega\right)^{2 \alpha_{i}}\right)^{\frac{\left(v_{i}-1\right)}{2 \alpha_{i}}},
$$

where $\eta_{0}$ is the zero-shear viscosity and $m$ is the number of curvatures in the shearthinning region. $\tau_{i}$ and $\alpha_{i}$ represent the characteristic viscous relaxation times and the width of each transition region between each curvature, respectively. $\left(\nu_{i}-1\right)$ is the slope $d \log \left|\eta^{*}\right| / d \log \omega$ of each curvature in the shear-thinning region.

This model describes the viscosity data as a sum of several simple generalized Newtonian fluid functions, whose individual behaviors are based on species exhibiting different relaxation times (i.e., different molecular architectures). Although the CY-Ext showed encouraging results, it is likely to present significant application restrictions when dynamic rheological analyses are considered. This extended model is based on the initial phenomenological CY model developed to describe steady-flow curves. Considering that $\eta(\dot{\gamma})$ and $\left|\eta^{*}(\omega)\right|$ differ significantly for branched polymers, data analysis may be inaccurate if the CY-Ext model is used to fit $\left|\eta^{*}(\omega)\right|$. Since the obtained curve is used to determine $w(\log m)$ from Eq. (8), discrepancies or loss of fundamental molecular structure-rheological information between calculation and experimental MWD are expected. The usefulness of the melt measurements to estimate MWDs is therefore questioned.

The procedure described in Sec. II could be extended to LCB polymers only if the LVE model for MWD calculation is independent of any empiricism and is defined as the fingerprint of the MWD. Various methods based on the "double reptation" mixing rule showed promising results to predict a quantitative relationship between LVE material functions and mono- or multimodal MWD. Mead (1994) and Wasserman (1995) developed analytical methods to invert the double reptation model in order to yield the underlying MWD. This technique is largely applied to deduce MWDs from LVE data, but the calculation procedure is a mathematically ill-posed problem and time consuming. The main difficulty arises from the inversion of Fredholm integral equations of the first kind. Any noise in the experimental data affects the precision of the results, and specific regularization techniques (e.g., Tikhonov regularization) are necessary to moderate these effects [Elster and Honerkamp (1992); Honerkamp and Weese (1993)].

Since MWD estimation procedure from melt measurements might be used regularly in industrial settings, experimentation and interpretation should be straightforward. Therefore, a simpler mathematical model which could be used together with the inversion procedure of Shaw and Tuminello (1994) and which contains essentially equivalent molecular information than the double reptation method is required.

Considering linear flexible polymers, Garcia-Franco and Mead (1999) revealed that the Cole-Cole model relaxation spectrum with appropriately chosen parameters is functionally similar to the double reptation model. In order to extend the empirical Cole-Cole model spectrum, initially introduced in the context of the dielectric relaxation, to the LVE behavior of polymer melts and specifically to $\left|\eta^{*}(\omega)\right|$, Havriliak and Negami (1967) proposed the generalization of the Cole-Cole function as follows:

$$
\eta^{*}(\omega)=\eta_{0}\left[1+\left(i \tau_{0} \omega\right)^{\alpha}\right]^{(\nu-1) / \alpha} .
$$


Using trigonometric identities and the scalar magnitude of Eq. (13) leads to the Havriliak-Negami model (referred to as HN)

$$
\left|\eta^{*}(\omega)\right|=\eta_{0}\left[1+2\left(\tau_{0} \omega\right)^{\alpha} \cos \left(\alpha \frac{\pi}{2}\right)+\left(\tau_{0} \omega\right)^{2 \alpha}\right]^{(\nu-1) / 2 \alpha},
$$

where $\eta_{0}$ is the zero-shear viscosity and $\tau_{0}$ is the characteristic viscous relaxation time. $\alpha$ is the width of the "knee" and $(\nu-1)$ is the high frequency slope $\mathrm{d} \log \left|\eta^{*}\right| / \mathrm{d} \log \omega$ of the shear-thinning region. This model has the great advantage to be exempted from the CoxMerz rule. However, values of $\alpha$ other than 1 cause an incorrect low frequency scaling. That is, $G^{\prime}$ and $G^{\prime \prime}$ do not have appropriate slopes of 2 and 1, respectively, on a logarithmic scale at low frequencies [Dorgan et al. (2005)].

The HN model is only useful to capture the main features of $\left|\eta^{*}(\omega)\right|$ data showing a unique curvature in the shear-thinning region and hence does not provide an accurate fitting in other cases. A complex viscosity model that captures the main features of $\left|\eta^{*}(\omega)\right|$ data showing a broad transition zone with several pronounced curvatures has not been described yet. Based on Eqs. (10) and (12), an extension of the Havriliak-Negami model (referred to as HN-Ext) is suggested in the present work as follows:

$$
\left|\eta^{*}(\omega)\right|=\eta_{0} \prod_{i=1}^{n}\left(1+2\left(\tau_{i} \omega\right)^{\alpha_{i}} \cos \left(\alpha_{i} \frac{\pi}{2}\right)+\left(\tau_{i} \omega\right)^{2 \alpha_{i}}\right)^{\frac{\left(v_{i}-1\right)}{2 \alpha_{i}}},
$$

where $n$ is the number of separated relaxation processes or curvatures in the shearthinning region. $\eta_{0}$ is the zero-shear viscosity value and $\tau_{i}$ is the characteristic viscous relaxation times of each transition region. $\alpha_{i}$ is the respective curvature of the knee and $\left(\nu_{i}-1\right)$ is the slope $\mathrm{d} \log \left|\eta^{*}\right| / \mathrm{d} \log \omega$ of each curvature in the shear-thinning region.

Equation (15) aims to fit available sets of $\left|\eta^{*}(\omega)\right|$ data corresponding to polymers featuring the above characteristics (i.e., broad transition zone with several curvatures). In other words, the double logarithmic slope $\mathrm{d} \log \left|\eta^{*}\right| / \mathrm{d} \log \omega$ must monotocally increase with decreasing $\omega$ (without local minimum in the investigated $\omega$ range), otherwise Eq. (15) cannot be used [Stadler and Munstedt (2008b)].

\section{MATERIALS AND METHODS}

\section{A. Materials}

Two different commercial PLA grades, named, PLA 4032D ${ }^{\circledR}$ and PLA 2002D ${ }^{\circledR}$, were purchased from NatureWorks (Arendonk, Belgium) [Ingeo-4032D (2005), Ingeo-2002D (2005)]. Technical specifications of both grades are given in Table I. A styrene-acrylic multifunctional-epoxide (referred to as SAmfE) oligomeric agent was used as reactive agent. It was kindly supplied by BASF (Ludwigshafen, Germany) in flake form under the commercial name Joncryl-ADR-4300F ${ }^{\circledR}$. The main characteristics of the SAmfE agent are summarized in Table II. Figure 1 shows its generic structure.

\section{B. One-step REX process}

REX was performed in a corotating twin screw extruder with a screw diameter of $25 \mathrm{~mm}(\mathrm{~L} / \mathrm{D}=36)$ (KNETER 25X24D, COLLIN, Ebersberg, Germany). Prior to processing, raw PLA pellets were dried for $3 \mathrm{~h}$ at $80^{\circ} \mathrm{C}$ in a PIOVAN hopper-dryer (DSN506HE, Venice, Italy) with a dew point of $-40^{\circ} \mathrm{C}$ and kept under the same 
TABLE I. Technical specifications for both PLA grades.

\begin{tabular}{|c|c|c|c|c|c|}
\hline PLA grade & $\begin{array}{c}\text { D-lactide } \\
\text { content }(\%)\end{array}$ & $\begin{array}{c}M_{n}^{\mathrm{a}} \\
\left(\mathrm{kg} \mathrm{mol}^{-1}\right)\end{array}$ & $\begin{array}{c}M_{w}{ }^{\mathrm{a}} \\
\left(\mathrm{kg} \mathrm{mol}^{-1}\right)\end{array}$ & $\begin{array}{c}M_{z}^{\mathrm{a}} \\
\left(\mathrm{kg} \mathrm{mol}^{-1}\right)\end{array}$ & $\begin{array}{c}\mathrm{MFI}^{\mathrm{b}} \\
(\mathrm{g} / 10 \mathrm{~min})\left(210^{\circ} \mathrm{C} / 2.16 \mathrm{~kg}\right)\end{array}$ \\
\hline Ingeo $4032 D^{\circledR}$ & 2 & 90 & 181 & 351 & $6.4 \pm 0.3$ \\
\hline Ingeo $2002 D^{\circledR}$ & 4.25 & 99 & 187 & 337 & $5.8 \pm 0.2$ \\
\hline
\end{tabular}

${ }^{a}$ Measured by SEC-DRI experiments (cf. Sec. IV C 1).

${ }^{\mathrm{b}}$ Measured by melt flow index experiments according to the ASTM D1238-04 C standard.

conditions during the whole process. SAmfE was powdered using mortar and pestle and vacuum-dried overnight at room temperature over silica gel.

It is well established that the reaction kinetics between hydroxyl groups and epoxy rings is notoriously slow [Bikiaris and Karayannidis (1996)]. Consequently, a maximum screw speed of $40 \mathrm{rpm}$ was chosen in order to maximize the residence time. In the current study, the maximum residence time of the extruder was $4.33 \mathrm{~min}$. The main emphasize of this work was a one-step REX-calendering process in a pilot plant.

Intermeshing corotating twin screws featuring three kneading blocks, each $100 \mathrm{~mm}$ in length, equally distributed along the screw length were used. The extruder features seven heating zones set at $150,160,160,160,170,175$, and $175^{\circ} \mathrm{C}$ from the feeding zone to the die, respectively. The extruder was operated by starve feeding at a constant rate of $2.54 \mathrm{~kg} \mathrm{~h}^{-1}$. Both polymer and SAmfE were simultaneously introduced in the hopper of the feeding zone under a nitrogen blanket ( 2.5 bars) after that the extrusion line was started and stabilized with raw PLA. The feeding rate of the SAmfE was adjusted to the polymer feeding rate in order to process each formulation described in Table III. Vacuum was applied in the metering zone to remove volatiles created during the reactive stage.

PLA sheets with a nominal thickness of $1 \mathrm{~mm}$ and a nominal width of $100 \mathrm{~mm}$ were calendered using a vertical three-polished chrome roller stack (Techline CR72T, COLLIN, Ebersberg, Germany). The chill roll $(\mathrm{D}=72 \mathrm{~mm})$ temperature was set to $50^{\circ} \mathrm{C}$ with a rotation speed of $1.25 \mathrm{rpm}$.

The melt pressure at the die, which is considered as a basic rheological response of the processed material, was monitored as function of extrusion time. The die pressure monotonously increased with extrusion time up to a constant value. At this point, no further increase in the die pressure was observed with the extrusion time, suggesting a stabilization of the rheological response of PLA to chain extension and/or branching reactions. Using the above described processing parameters, about $20 \mathrm{~m}$ of calendered sheet were obtained for each formulation.

Accordingly, samples prior to the SAmfE addition (referred to as PLA4 and PLA2) and from the stabilized die pressure regime were analyzed (referred to as PLA4-REX0.5, PLA2-REX0.5, and PLA2-REX0.6). All prepared samples are summarized in Table III; samples were distinguished by their different D-lactide content and the nominal amount of added SAmfE.

TABLE II. Technical specifications for SAmfE agent.

Functionally: $\approx 12$

Glass transition temperature: $56^{\circ} \mathrm{C}$

MW: $5443 \mathrm{~g} \mathrm{~mol}^{-1}$

Epoxy equivalent weight: $433 \mathrm{~g} \mathrm{~mol}^{-1}$ 


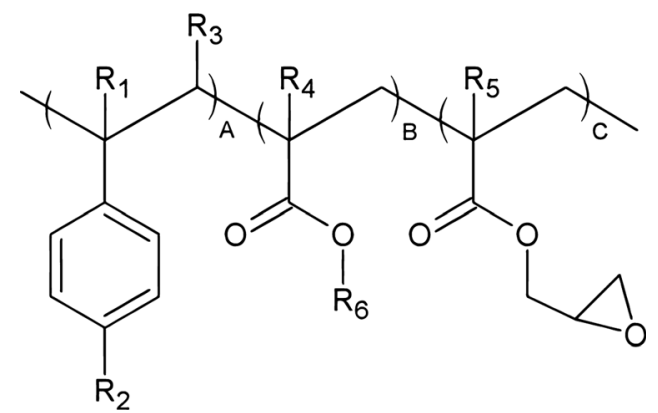

FIG. 1. Structure of SAmfE agent. $\mathrm{R}_{1}-\mathrm{R}_{5}$ are $\mathrm{H}, \mathrm{CH}_{3}$, a higher alkyl group, or combinations of them; $\mathrm{R}_{6}$ is an alkyl group, and $\mathrm{A}$ and $\mathrm{B}$ are each between 1 and 20 and $\mathrm{C}$ between 1 and 12 [based on Joncryl-ADR-4300 (2006)].

\section{Characterization techniques}

\section{SEC}

MWDs were measured on an Agilent HPLC model Infinity 1260 SEC system (Agilent Technologies, Santa Clara, CA) with differential refractive index detection (SEC-DRI) at room temperature. Filtered samples were injected at a volume of $100 \mu \mathrm{l}$. An Agilent PL HFIPgel $(300 \times 7.5 \mathrm{~mm})$ specialist column with a separation range from 200 to $800 \mathrm{~kg} \cdot \mathrm{mol}^{-1}$ was used at a flow rate of $1 \mathrm{ml} \mathrm{min}^{-1}$. Hexafluoroisopropanol $(1,1,1,3,3,3$ hexafluoro-2-propanol, HFIP) containing $0.02 \mathrm{~mol}^{-1}$ of sodium trifluoroacetate to prevent polyelectrolyte effect was used as eluent. Measurements were repeated three times using fresh samples in order to improve the accuracy. A total of seven narrow poly(methyl methacrylate) standards with MWs covering the entire MW range of the investigated samples (from 2 to $900 \mathrm{~kg} \mathrm{~mol}^{-1}$ ) were injected to perform the $\log M_{w}$ versus retention time calibration via the peak position method [Wu (2003)].

\section{Small amplitude oscillatory shear measurements}

Prior to testing, amorphous samples were prepared in order to enlarge the temperature window for dynamic measurements. In a typical procedure, samples were vacuum-dried over silica gel at $55^{\circ} \mathrm{C}$ overnight and melted at $5{ }^{\circ} \mathrm{C}$ above their respective melting temperature for $3 \mathrm{~min}$ between two steel plates in a hot plate press. Then, samples were quickly quenched in an ice water bath $\left(2 \pm 2{ }^{\circ} \mathrm{C}\right)$ for $5 \mathrm{~min}$ in order to avoid crystallization. After drying, disks with $25 \mathrm{~mm}$ in diameter were extracted from the center of the sheet and vacuum-dried overnight at room temperature over silica gel. The amorphous state of samples was investigated using differential scanning calorimetry experiments, from the first heating run $\left(30-200^{\circ} \mathrm{C}\right)$ at $10^{\circ} \mathrm{C} \mathrm{min}^{-1}$. As it will be explained in Sec. VC, solely unmodified PLA samples (i.e., PLA4 and PLA2) were stabilized with an

TABLE III. Formulation of all investigated samples.

\begin{tabular}{lccc}
\hline \hline PLA grade & Sample nomenclature & Neat polymer content (wt. \%) & Nominal SamfE amount (wt. \%) \\
\hline Ingeo 2002D & PLA4 & 100 & 0 \\
& PLA4-REX0.5 & 99.5 & 0.5 \\
Ingeo 4032D & PLA2 & 100 & 0 \\
& PLA2-REX0.5 & 99.5 & 0.5 \\
& PLA2-REX0.6 & 99.4 & 0.6 \\
\hline \hline
\end{tabular}


antioxidative stabilizer Irganox 1010 (1 wt. \%) in order to avoid excessive thermal degradation throughout rheological measurements.

Measurements were performed on an AR-G2 magnetic bearing rheometer (TA Instruments, New Castle, DE) in parallel plate $(25 \mathrm{~mm})$ configuration with a constant gap of $1 \mathrm{~mm}$. Tests were conducted in dry nitrogen atmosphere in order to minimize the thermo-oxidative degradations of the resin. The melt thermal stability throughout rheological measurements was verified through dynamic time sweep experiments at $50 \mathrm{rad}$ $\mathrm{s}^{-1}$ with $0.2 \%$ strain amplitude. Dynamic strain sweeps defined the LVE regime. Dynamic frequency sweeps over an angular frequency $\omega$ from 628 to $0.0628 \mathrm{rad} \mathrm{s}^{-1}$ were performed at different temperatures $\left(160-170-180-200^{\circ} \mathrm{C}\right)$ and at fixed strain amplitude in the LVE. Each test was repeated three times using fresh samples. The technique developed by Tchir and Saucier (1994) was used to minimize the between-sample error. The viscoelastic functions measured at different temperatures were reduced to master curves at $180^{\circ} \mathrm{C}$ using the TTS principle for samples which relaxation processes are not time-dependent with temperature (thermorheologically simple). This temperature $\left(180^{\circ} \mathrm{C}\right)$ was chosen due to the better compromise between flow properties and thermal stability throughout rheological testing.

In order to ensure sufficient data at low $\omega$, creep-recovery tests were conducted at $180^{\circ} \mathrm{C}$. Within the LVE, the creep time was set to be sufficiently long to reach the steady state according to the methodology of Gabriel and Munstedt (1999). The recovery experiments were started from the steady state of the creep phase and were conducted until reaching a steady value of recoverable compliance. The average of three fresh samples was determined. Creep-recovery data were converted to retardation spectra $L(\lambda)$ using the commercial RHEOLOGY ADVANTAGE software (TA Instruments) based on the NLREG method developed by Honerkamp and Weese (1993). Then the complex shear compliance $J^{*}(\omega)$ was calculated from $L(\lambda)$ and $\left|\eta^{*}(\omega)\right|$ from $J^{*}(\omega)$.

\section{NMR}

${ }^{1} \mathrm{H}$ NMR spectra were obtained using a Bruker AMX spectrometer (Bruker Corporation, Billerica, MA) operating at $300 \mathrm{MHz}$ for proton investigation with tetramethylsilane as internal standard. Prior to testing, samples were dissolved in a deuterochloroform solution $\left(\mathrm{CDCl}_{3}\right)$ with a concentration of $30 \mathrm{mg} \mathrm{ml}^{-1}$. A total of 64 scans with $32 \mathrm{k}$ data points were recorded with a relaxation delay of $1 \mathrm{~s}$.

\section{RESULTS AND DISCUSSION}

\section{A. MW characterization}

The multifunctional epoxy-functionalized reactive agent, used in manufacturing PLAREX samples, is able to promote the formation of LCB and/or complex topological structures, as shown elsewhere [Corre et al. (2011); Najafi et al. (2012); Meng et al. (2012); Al-Itry et al. (2014)]. In solution, LCB polymers exhibit a more compact configuration than their linear homologues for a given MW. An underestimation of the MW of LCB polymers by SEC is likely to occur due to coelution issues of different molecular topologies. For the purpose to provide the closest approximation to the real MWD for branched polymers, SEC coupled with multiple detectors should be used. A preliminary trial was performed to evaluate the MW of PLA-REX samples using SEC with in line DRI, viscometry, and multiangle LS (MALLS) detectors. Before testing, samples were carefully dissolved in tetrahydrofuran (THF, used as eluting phase) and filtered. After filtering, the filters were dried under vacuum, in order to remove the remaining THF, and subsequently 
weighted to contribute to a quantitative detection of possible gel structures. The weight of the filters before and after filtration was found to be roughly similar, confirming the total dissolution of all the samples in THF. Nevertheless, the radius of gyration (as measured by MALLS) as well as the intrinsic viscosity (as measured by viscometer) was found to be roughly similar for all samples, indicating the absence of structural changes. As will be shown in Sec. VD, significant differences in rheological behavior of the different samples were found. Therefore, it can be assumed that the aforementioned SECmultiple detectors results are not trustworthy.

Similar behavior have been already reported for investigating sparsely LCB-PE [Wang et al. (2004)], polypropylene featuring low amounts of LCB [Lagendijk et al. (2001); Auhl et al. (2004)], and for LCB metalocene catalyzed PE (which is a blend of linear and branched chains) [Stadler et al. (2006)]. Authors concluded that this technique was not enough sensitive to estimate slight changes in molecular dimensions between the very low amount of nonuniform branched structures and the dominant linear fraction. While we could expect that SEC-DRI data may be flawed due to coelution issues, van Ruymbeke et al. (2005) reported that the error on the MWD may be considered to be negligible for low levels of structural modifications. On this basis, standard SEC-DRI equipment was preferred to investigate the molecular modifications of PLA induced by REX. Figure 2 shows the MWD for PLA and PLA-REX samples, and the corresponding relative MW value averages are compiled in Table IV.

While both PLA samples displayed a narrow monomodal MWD, PLA-REX spectra were broadened and a shoulder appeared in the HMW tail. Results suggest that the two principal hydrodynamic volumes, which controlled the elution time, were roughly similar between PLA-REX samples since all three spectra were approximately centerd on the same MW peak values. The displacement of the original PLA peak to higher MWs implies that weight fractions with higher MWs were generated at the expense of the low MW populations.

Since SAmfE is a multifunctional-epoxide reactive agent, the observed trend could be attributed to the chemical bonding of several PLA chains to one SAmfE molecule. However, the topological modifications achieved are not trivial and would be a mixture of several types of nonuniform structures in MW, in architectures and in the number of branch per macromolecules. During sample conditioning, the total dissolution of the samples in HFIP confirmed that no gels were generated. In this way, differences in the MWD

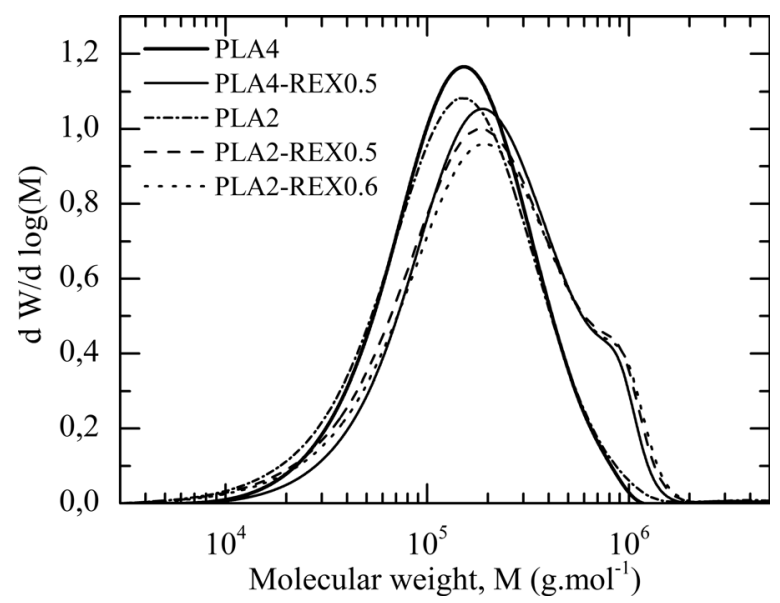

FIG. 2. Effect of the reactive process on the shape of the MWDs. 
TABLE IV. Relative MW value averages.

\begin{tabular}{lcccc}
\hline \hline Sample nomenclature & $M_{n}{ }^{\mathrm{a}}\left(\mathrm{kg} \mathrm{mol}^{-1}\right)$ & $M_{w}{ }^{\mathrm{a}}\left(\mathrm{kg} \mathrm{mol}^{-1}\right)$ & $M_{z}{ }^{\mathrm{a}}\left(\mathrm{kg} \mathrm{mol}^{-1}\right)$ & $M_{w} / M_{n}{ }^{\mathrm{a}}$ \\
\hline PLA4 & $92 \pm 6$ & $185 \pm 3$ & $329 \pm 30$ & $2.0 \pm 0.2$ \\
PLA4-REX0.5 & $119 \pm 1$ & $246 \pm 2$ & $475 \pm 7$ & $2.08 \pm 0.03$ \\
PLA2 & $85 \pm 1$ & $179 \pm 1$ & $345 \pm 4$ & $2.13 \pm 0.04$ \\
PLA2-REX0.5 & $106 \pm 2$ & $272 \pm 3$ & $545 \pm 10$ & $2.57 \pm 0.08$ \\
PLA2-REX0.6 & $120 \pm 1$ & $292 \pm 1$ & $604 \pm 83$ & $2.44 \pm 0.03$ \\
\hline \hline
\end{tabular}

${ }^{a}$ Relative MW values were calculated from the average of three experiments based on fresh samples.

shape may indicate an increase in the population of HMW linear chains and/or the presence of branched structures. This hypothesis is in line with the significant enhancement of MWs between PLA and all PLA-REX samples and with the study of Corre et al. (2011).

It is well known that the inverse of the number-average MW $\left(M_{n}\right)$ is related to the number of functional terminal groups and $M_{n}$ to the number of entanglements per chains. As can be seen in Table IV, $M_{n}$ is increased with the addition of SAmfE, suggesting the consumption of terminal end groups during chain extension and/or chain branching reactions and an increase in the number of entanglements per chains.

PLA2-REX0.5 samples exhibited higher MW values than PLA4-REX0.5 samples for equivalent amount of SAmfE agent. This trend may be due to the combination of two different effects. On one hand, PLA2 raw material shows a higher amount of functional end groups than PLA4 one, which could result in a higher reactivity toward SAmfE during processing. On the other hand, the degree of thermal degradation during processing should be considered, as terminal end groups for commercial PLA are usually end capped [Bigg (2005); Liu et al. (2010)]. Bigg (2005) suggests that the thermal stability during processing depend on the width of the processing windows. As already reported by Cailloux et al. (2013), PLA2 exhibited a narrower processing window than PLA4 material (according to their respective end melting temperatures), which aspect will increase the local shear field applied to the material during mixing and could increase the thermomechanical degradation effects. Therefore, a higher amount of reactive functional end groups may be created, and the reactivity toward SAmfE was probably enhanced.

\section{B. Melt stability throughout rheological testing}

Dynamic time sweep experiments were conducted during $1450 \mathrm{~s}(\approx 24 \mathrm{~min})$ in order to ensure the melt stability throughout rheological measurements. This experimental time allows to complete both frequency sweeps over an angular frequency ranged from 628 to $0.0628 \mathrm{rad} \mathrm{s}^{-1}$ with ten points per decade and creep-recovery experiments (using fresh samples). Although the melt stability was evaluated for each testing temperature, only results corresponding to the measurements at $200^{\circ} \mathrm{C}$ were plotted in Fig. 3. This temperature was chosen due to the usual poor thermal stability of PLA at high temperature over prolonged time (see Fig. 3, unstabilized PLA4 sample).

The addition of either $1 \mathrm{wt}$ \% of Irganox 1010 or the addition of SAmfE significantly increased the melt stability of both PLA types. Al-Itry et al. (2014) suggested that thermal degradation of PLA can be assumed as negligible if the complex viscosity, normalized by its initial values at $t=0\left(\left|\eta^{*}\right| /\left|\eta^{*}(t=0)\right|\right)$ does not decrease by more than $10 \%$ within the total testing time. As shown in Fig. 3, the $\left|\eta^{*}\right| /\left|\eta^{*}(t=0)\right|$ traces slightly decreased and increased $( \pm 8 \%)$ over testing time for PLA and PLA-REX samples, respectively. 


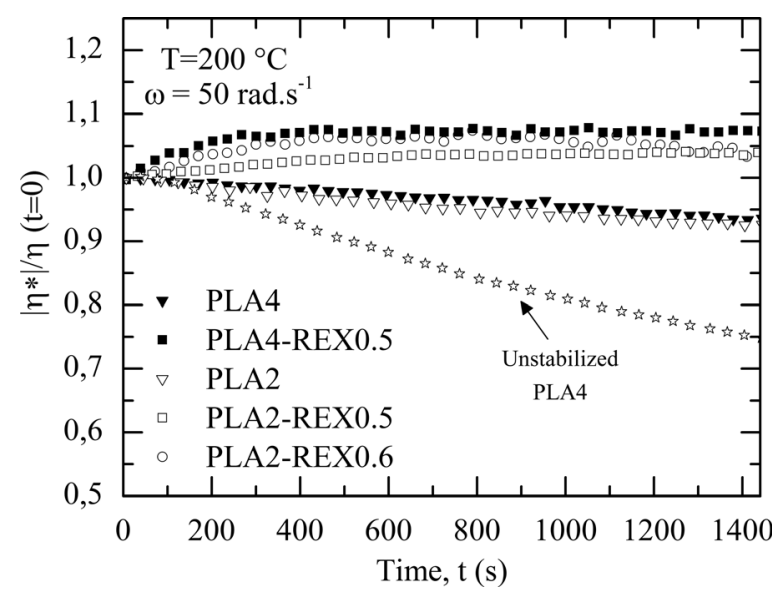

FIG. 3. Thermal stability of PLA and PLA-REX samples at $200{ }^{\circ} \mathrm{C}$ under a nitrogen atmosphere at $50 \mathrm{rad} \mathrm{s}^{-1}$.

In order to determine if the MWD and MW parameters of the samples showed modifications after rheological testing, the MW was evaluated using SEC-DRI on the recovered samples. The total dissolution of the samples in HFIP suggested that no gels were generated during rheological measurements. Although not shown in this communications, the MWD traces were found to be roughly similar to that shown in Fig. 2 and the relative MW averages and PDI values agreed with previous MW values (see Table IV) within $10 \%$. Consequently, these trends suggest that further rheological properties remained fairly unaffected by thermal degradations and/or MW changes throughout rheological testing.

\section{Investigation of the temperature dependence}

Special care should be taken when operators are working with the TTS principle. In order to generate the final master curve over an extended range of $\omega$, the considered material has to present a thermorheologically simple behavior. The essence of this concept is based on the similar modification of the time dependence of molecular motions and of relaxation times by changing the temperature of measurements.

Within the LVE regime, the vGP plot is a useful tool for detecting thermorheological simplicity or complexity behavior [Van Gurp and Palmen (1996)]. This powerful way of verifying the success of application of the TTS principle is based on the exclusion of temperature and frequency dependence for well entangled polymers [Dealy and Larson (2006c); Trinkle and Friedrich (2001); Dealy and Larson (2006a)]. As shown in Fig. 4, the phase angle $\delta=\arctan$ (loss modulus $\left(G^{\prime \prime}\right)$ /storage modulus $\left(G^{\prime}\right)$ ) of several frequency sweeps performed at different temperatures is plotted against the absolute value of the corresponding complex modulus, $\left|G^{*}\right|$. Regardless of the polymer tacticity or chemical nature, all data should merge into a same curve if the TTS holds.

PLA4 and PLA2 data are shown in the same plot due to the similar shape and temperature dependence observed for $\delta$ as a function of $\left|G^{*}\right|$. According to the above vGP criterion, PLA4, PLA2, PLA4-REX0.5, and PLA2-REX0.5 samples could be considered as thermorheologically simple. At first glance, a modification of the dependence of $\delta$ on $\left|G^{*}\right|$ values for PLA4-REX0.5 and PLA2-REX0.5 as compared to their linear counterparts was found. This trend could be attributed to a modification of the molecular 
architecture (i.e., chain extension/branching) through REX. On the other hand, PLA2REX0.6 samples were thermorheologically complex. This loss of thermorheological simple behavior suggests the formation of topological modifications, which probably hold LCB, as already reported by Al-Itry et al. (2014) for PLA and Wood-Adams and Costeux (2001), Lohse et al. (2002), and van Ruymbeke et al. (2005) for LCB polyolefins. That is, the presence of long chain branches significantly increases the melt response time and commonly yields a modification of the sequence of the molecular relaxations as function of the testing temperature. In the present work, the architectural changes achieved are probably not well defined as PLA2-REX0.6 samples are not a carefully prepared model polymer based on specific polymerization technologies.

For the purpose to evaluate the effects of both the stereoregularity and the topological modifications on the temperature dependence of the viscoelastic functions, the activation energy, $E_{a}$, was first investigated. Recall that the concept of a single activation energy is meaningless if the temperature sensitivity is time dependent. Consequently, $E_{a}$ was solely calculated for thermorheologically simple samples. In both the frequency range and the temperature experimental windows evaluated, $\eta_{0}$ was not straightforwardly observed for PLA4-REX0.5 and PLA2-REX0.5 samples. Thus, $G^{\prime}(\omega)$ was considered a better candidate for the shifting process. The $G^{\prime}$ curves, measured at 160,170 , and $200{ }^{\circ} \mathrm{C}$, were shifted along the frequency axis to $180^{\circ} \mathrm{C}$ using the TTS principle. Since the viscoelastic measurements were available only far above the glass transition temperature of all sample $\left(T_{g} \approx 61^{\circ} \mathrm{C}\right)$ and over a relatively narrow temperature range, the horizontal shift factor, $a_{T}$, follows an Arrhenius relation with temperature, as follows [Dealy and Larson (2006b)]:

$$
a_{T}(T)=\exp \left[\frac{E_{a}}{R}\left(\frac{1}{T}-\frac{1}{T_{0}}\right)\right],
$$

where $R$ is the gas constant. $T$ and $T_{o}$ are the temperature of measurement and of reference, respectively.

$E_{a}$ was obtained from the best linear regression of a set of values represented in a graph plotting the natural logarithmic values of $a_{T}$ versus $1 / T$. Over the range of data considered, $E_{a}$ did not show a clear dependence neither on the stereoregularity between both linear PLA samples nor on the architectural modifications between PLA4-REX0.5, PLA2-REX0.5 samples, and their linear homologues. Close examination of the $E_{a}$ measurements revealed that all values ranged from 85 to $88 \mathrm{~kJ} \mathrm{~mol}^{-1}$ within the statistical error of the experiments.

These results are consistent with the modest effects of the chain architectures on the flow activation values (ranged between 79 and $116 \mathrm{~kJ} \mathrm{~mol}^{-1}$ ) reported by Dorgan et al. (1999) for linear and branched (i.e., star-shaped) PLA polymers. Authors stated that differences in the PLA molecular architecture cannot be distinguished using the calculation of $E_{a}$, because the temperature dependency of the viscoelastic functions is independent of the MW but mainly depends on local segmental relaxations rather than large-scale relaxation mechanisms (e.g., reptation, constraint release, etc.).

The similarity of $E_{a}$ values, independently of the chain architectures, suggests that this technique is not enough sensitive to distinguish subtle differences in the PLA molecular architecture. However, the absence of an enhanced $E_{a}$ and/or the loss of thermorheological simple behavior does not necessarily exclude the presence of topological changes in PLAREX samples, which possibly hold low levels of LCB, as already reported in Wasserman and Graessley (1996), Vega et al. (1998), and Shroff and Mavridis (1999) for sparsely LCB HDPE resins and Munari et al. (1990) for branched poly(butyleneisophthalate). 

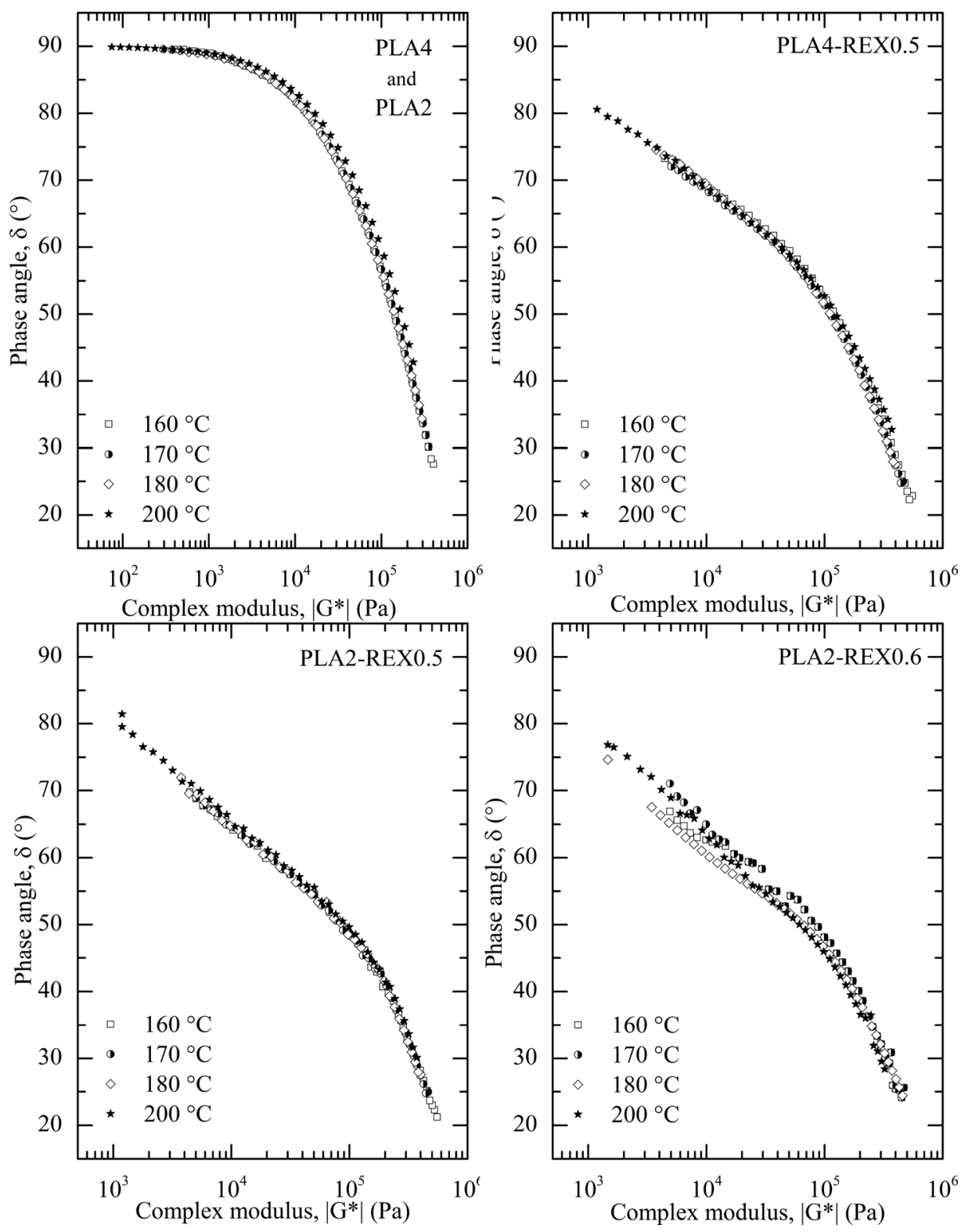

FIG. 4. Verification of the TTS principle for all investigated samples.

\section{Effect of the topological changes on the viscoelastic properties}

In order to determine if PLA4-REX0.5 and PLA2-REX0.5 samples showed any behavior consistence with the presence of topological modifications, which might hold low amounts of LCB, the viscoelastic functions were investigated. The storage modulus master curves, $G^{\prime}(\omega)$, which are obtained from frequency sweep and creep-recovery experiments of thermorheologically simple samples (i.e., PLA2, PLA4, PLA4-REX0.5, PLA2-REX0.5) are shown in Fig. 5. This dynamic mechanical spectrum, which covers seven magnitudes of angular frequency, is the combination of two type of experiments: (a) Data in the range $10^{-1}-10^{4} \mathrm{rad} \mathrm{s}^{-1}$ obtained from the TTS and (b) data in the range 
$5 \times 10^{-4}-10^{-1} \mathrm{rad} \mathrm{s}^{-1}$ obtained from the conversion of the creep-recovery data by the commercial RHEOLOGY ADVANTAGe software. On the other hand, $G^{\prime}(\omega)$ of PLA2-REX0.6 was added to Fig. 5. It was solely constructed from the combination of the frequency sweep performed at $180^{\circ} \mathrm{C}$ and the conversion of the creep-recovery data, as this sample exhibited a thermorheologically complex behavior.

As shown in Fig. 5, both parts connected well with each other and showed different relaxation information between PLA-REX samples and their linear homologues. In the high $\omega$ range $\left(10^{3}-10^{4} \mathrm{rad} \mathrm{s}^{-1}\right), G^{\prime}$ values converged toward a roughly similar value. This behavior suggests that the molecular motions, which govern the high $\omega$ range, remained independent of the molecular structure, as already reported in Kasehagen et al. (1996) and Dealy et al. (2006).

In contrast, significant differences in $G^{\prime}(\omega)$ were observed in the low frequency range $\left(10^{-3}-10^{2} \mathrm{rad} \mathrm{s}^{-1}\right)$. An additional intermediate relaxation regime (indicated by an arrow) was observed between the terminal (i.e., $G^{\prime} \propto \omega^{2}$ ) and plateau region for PLA-REX samples. Moreover, higher and increasing $G^{\prime}$ values were found in the low $\omega$ range for both the more reactive PLA type (i.e., PLA2) and the increasing SAmfE content. Results suggest the formation of a network having a higher melt strength and which is less shear sensitive with decreasing $\omega$ [Liu et al. (2010); Corre et al. (2011)]. Nevertheless, despite the significant differences observed in $G^{\prime}(\omega)$ between PLA-REX samples and their linear homologues, conclusions about the presence of LCB cannot be drawn from Fig. 5. This is because both the increase in MW as measured by SEC-DRI (cf. Table IV) and the topological modifications have convoluted effects on the dependence of $G^{\prime}$ on $\omega$.

Similarly, $\left|\eta^{*}(\omega)\right|$ master curves, which were constructed from the combination of the TTS and the conversion of creep-recovery data for thermorheologically simple samples (i.e., PLA2, PLA4, PLA4-REX0.5, and PLA2-REX0.5), are shown in Fig. 6. $\left|\eta^{*}(\omega)\right|$ of PLA2-REX0.6 was also added to Fig. 6, but it was derived solely from the combination of the frequency sweep performed at $180{ }^{\circ} \mathrm{C}$ and the conversion of the creep-recovery data (thermorheologically complex behavior).

Both PLA samples (i.e., PLA4 and PLA2) exhibited the typical behavior of linear polymers. The terminal (Newtonian) region was observed over a wide range of $\omega$ and was followed by the pseudoplastic region with increasing $\omega$. In contrast, besides a significant increase in $\eta_{0}$, the onset of the terminal region was shifted to lower $\omega$ for PLA-REX

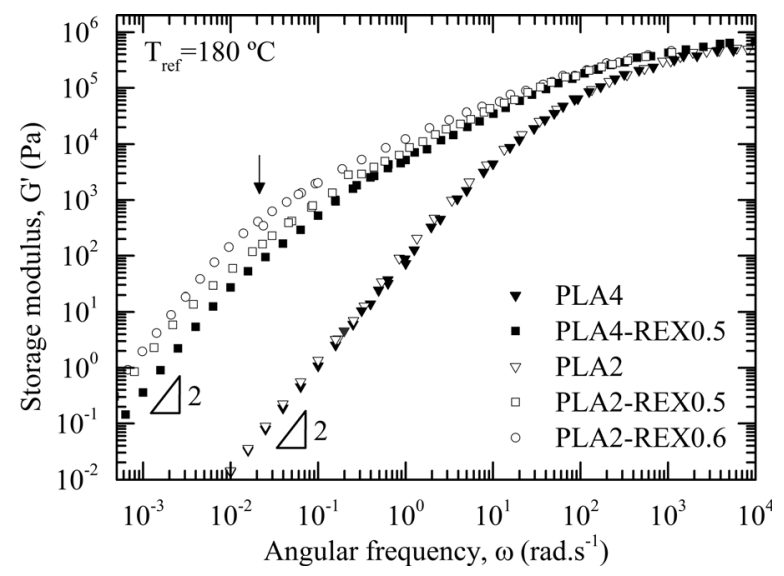

FIG. 5. Influence of the reactive process on the storage modulus function at $180^{\circ} \mathrm{C}$. The arrow indicates an additional curvature in the transition zone between the terminal and the plateau region in $G^{\prime}(\omega)$. 


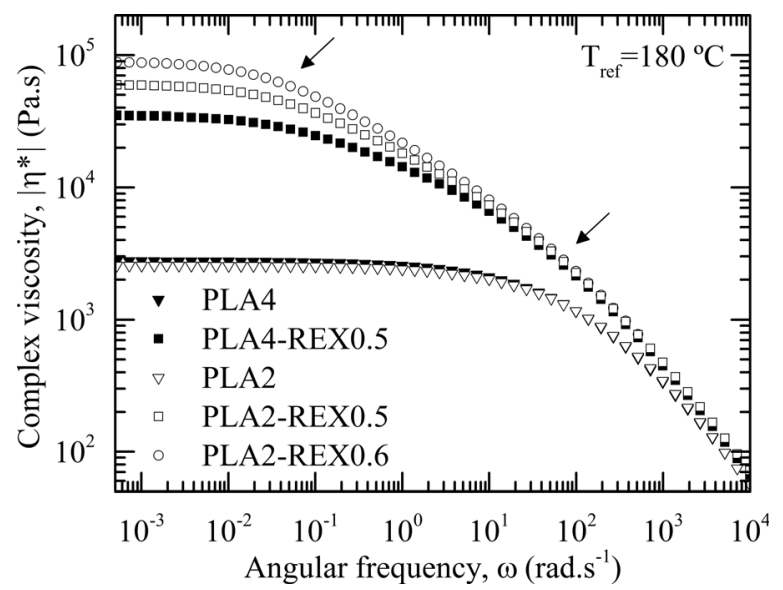

FIG. 6. Effect of the addition of the SAmfE agent on the absolute magnitude of the complex viscosity versus angular frequencies at $180^{\circ} \mathrm{C}$. The arrows indicate two different curvatures in the transition zone between the terminal and power-law region in $\left|\eta^{*}(\omega)\right|$.

samples. The transition zone between the terminal and power-law region was enlarged, and two different curvatures were observed (indicated by arrows).

The CY [Eq. (11)] and HN [Eq. (14)] models were used to fit $\left|\eta^{*}(\omega)\right|$ experimental data of PLA4 and PLA2 samples. Both functions were fitted to the entire set of available data using the least square method in order to capture the salient feature of $\left|\eta^{*}(\omega)\right|$ in an efficient and accurate numeric way. The resulting parameters are given in Table V. Figure 7 shows the double logarithmic slope $\mathrm{d} \log \left|\eta^{*}\right| / \mathrm{d} \log \omega$ in the investigated $\omega$ range for PLA-REX samples.

Since $\mathrm{d} \log \left|\eta^{*}\right| / \mathrm{d} \log \omega$ monotonously increased with decreasing $\omega$, both the CY-Ext [Eq. (12)] and the HN-Ext [Eq. (15)] models were used to fit $\left|\eta^{*}(\omega)\right|$ data with $m=n=2$ due to the double curvature observed in the transition zone (cf. Fig. 6). Table V summarizes the parameters of the different fits.

As shown in Table V, the first characteristic relaxation time $\left(\tau_{1}\right)$ increased with both the more reactive PLA type (i.e., PLA2) and the SAmfE content. These results suggest that the transition zone between the Newtonian and the power-law region was broadened with an increase in $M_{w}$ (cf. Table IV). In addition, the increasing values of $\nu_{2}$ imply that $\left|\eta^{*}(\omega)\right|$ was more shear-sensitive with increasing $M_{w}$. Although these trends could be attributed to the alteration of the molecular mobility due to the increasing number of interactions and entanglements per chains (i.e., higher relative $M_{n}$ values for PLA-REX samples, cf. Table IV), both the increase in MW and the architectural modifications have similar effects on the viscosity function. One of the most prominent differences was the increase in MW according to SEC-DRI measurements (cf. Table IV).

Therefore, an appropriate method to remove the MW dependence on the $\left|\eta^{*}(\omega)\right|$ behavior is looked for. One of the most useful methods is the time-MW (TMW) superposition principle, which is fulfilled for polymer exhibiting similar and narrow MWD (e.g., PLA and PLA-REX samples, cf. Table IV) [Doerpinghaus and Baird (2003)]. Initially, this concept assumes that the dependence of $\eta_{0}$ on $M_{w}$ follows Eq. (3b) for linear polymer well above $M_{c}$. Then, Ferry (1980) reported that the longest relaxation time, $\tau_{1}$, was proportional to the product of the steady-state compliance, $J_{e}{ }^{0}$ and $\eta_{0}$, as given by

$$
\tau_{1} \propto J_{e}^{0} \eta_{0} .
$$


TABLE V. Numerical parameters of the different fitting models at $180^{\circ} \mathrm{C}$.

\begin{tabular}{|c|c|c|c|c|c|c|c|c|}
\hline \multirow[b]{2}{*}{ Fitting models ${ }^{\mathrm{a}}$} & \multicolumn{2}{|c|}{ PLA4/PLA2 } & \multicolumn{2}{|c|}{ PLA4-REX0.5 } & \multicolumn{2}{|c|}{ PLA2-REX0.5 } & \multicolumn{2}{|c|}{ PLA2-REX0.6 } \\
\hline & $\mathrm{CY}$ & $\mathrm{HN}$ & CY-Ext & HN-Ext & CY-Ext & HN-Ext & CY-Ext & HN-Ext \\
\hline$\eta_{0}(\mathrm{kPa} \mathrm{s})$ & $2.8 / 2.6$ & $2.8 / 2.6$ & 35.4 & 35.4 & 60.1 & 60.1 & 90 & 90 \\
\hline$\tau_{1}(\mathrm{~s})$ & $\begin{array}{l}5.19 \times 10^{-3} \\
/ 4.51 \times 10^{-3}\end{array}$ & $\begin{array}{c}5.19 \times 10^{-3} \\
/ 4.51 \times 10^{-3}\end{array}$ & 29.85 & 29.85 & 35.60 & 35.60 & 42.85 & 42.85 \\
\hline$\alpha_{1}$ & $0.55 / 0.59$ & $0.50 / 0.53$ & 1 & 1 & 1 & 1 & 1 & 1 \\
\hline$\nu_{1}$ & $0.0726 / 0.038$ & $0.0726 / 0.038$ & 0.795 & 0.795 & 0.708 & 0.708 & 0.673 & 0.673 \\
\hline$\tau_{2}(\mathrm{~s})$ & - & - & 0.01 & 0.01 & 0.0065 & 0.0065 & 0.006 & 0.006 \\
\hline$\alpha_{2}$ & - & - & 0.45 & 0.381 & 0.45 & 0.38 & 0.41 & 0.36 \\
\hline$\nu_{2}$ & - & - & 0.240 & 0.240 & 0.332 & 0.332 & 0.362 & 0.362 \\
\hline
\end{tabular}

${ }^{\mathrm{a} C Y}$ : Carreau-Yassuda, HN: Havriliak-Negami, CY-Ext: Extended Carreau-Yassuda, HN-Ext: Extended Havriliak-Negami.

Since Ferry (1980) reported that $J_{e}{ }^{0}$ is commonly found to be independent of $M_{w}$ above $2 M_{c}$, the resulting relationship for the longest relaxation time yields the same $M_{w}$ dependence as $\eta_{0}$. Consequently, when these concepts are combined, an equivalent shifting along the time, or rate, axis and the viscosity axis is achieved. On this basis, the TMW superposition is applied using the shift factor, $a_{M}$, as follows [Doerpinghaus and Baird (2003)]:

$$
a_{M}=\frac{\eta_{0}\left(M_{w}\right)}{\eta_{0}\left(M_{w}\right)_{L}},
$$

where $\eta_{o}\left(M_{w}\right)$ is the zero-shear rate viscosity of the modified samples (e.g., PLA-REX) and $\eta_{0}\left(M_{w}\right)_{L}$ is the zero-shear rate viscosity of its corresponding linear samples of equivalent MW. In principle, the application of the shift factor, $a_{M}$, should reveal the individual contribution of the topological modifications on the $\left|\eta^{*}(\omega)\right|$ function [Doerpinghaus and Baird (2003)].

For the purpose to remove the MW effects of the viscosity functions of PLA-REX samples, the established dependence of $\eta_{0}$ on $M_{w}$ for linear polydisperse PLA at $180^{\circ} \mathrm{C}$ [i.e., $\eta_{0}=5.5 \times 10^{-15} M_{w}^{3.4}$, Dorgan et al. (2005)] is used to estimate $\eta_{0}$ of their corresponding linear sample of equivalent MW. The shifted viscosity curves are shown in Fig. 8 together with both linear PLA samples.

As shown in Fig. 8, a difference in the dependence of $\left|\eta^{*}\right|$ on $\omega$ between PLA and PLA-REX samples is apparent. While $\eta_{0}$ increased, the onset of the terminal region was shifted to lower $\omega$ and the transition zone between the Newtonian and the power-law region was enhanced for the latter. Moreover, the additional curvature between both regions was still exhibited by PLA-REX samples. These trends are one of the noteworthy effects of the alteration of the molecular mobility due to the increasing number of interactions and entanglements per chains (i.e., higher relative $M_{n}$ values for PLA-REX samples, cf. Table IV), which led to a decreasing rate of molecular relaxation. It could occur when the polymeric chains, belonging to the architecturally modified macromolecules, become sufficiently long to entangle and impede the segmental motions of the neighboring chains more strongly than simple hydrodynamic drag. Since the TMW principle shifts rheological data to a single master curve if all the considered samples are linear, the above results suggest the presence of macromolecules featuring longer relaxation times. In other words, these differences in the trend of $\left|\eta^{*}(\omega)\right|$ must be primarily attributed to the 


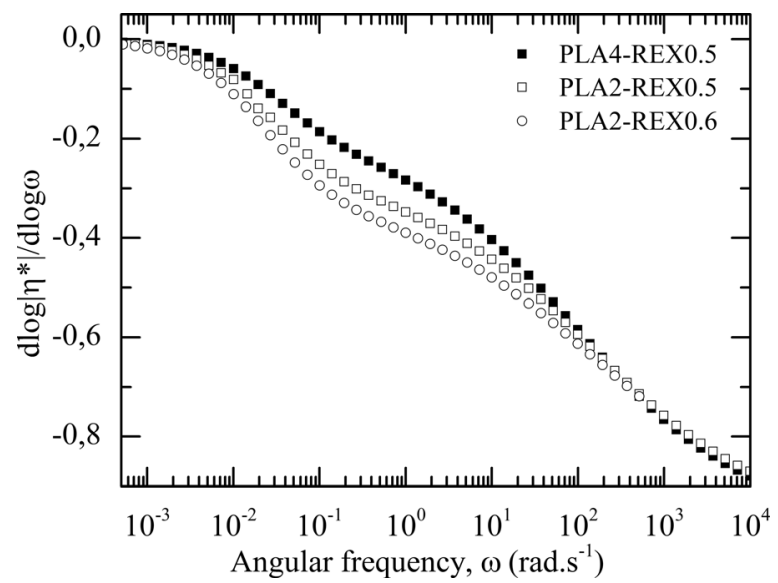

FIG. 7. Double logarithmic derivative of the PLA-REX $\left|\eta^{*}(\omega)\right|$ functions.

presence of different branched structures which possibly hold low levels of long chain branches [Stadler and Munstedt (2008a); Lohse et al. (2002)] rather than the minor variations in the PDI values (cf. Table IV). On the other hand, $\eta_{0}$ gradually shifted to higher values for both the more reactive PLA type (i.e., PLA2) and the increasing SAmfE content, which behavior might be correlated with an increasing amount of modified PLA chains. This is further supported by the fact that the trend of temperature dependence of the viscoelastic functions changed from a thermorheological simple to a complex behavior with increasing the SAmfE content (cf. Fig. 4, PLA2-REX0.5 and PLA2-REX0.6 samples).

To further strengthen the assumption that PLA-REX samples possibly hold LCB, the experimental $G^{\prime}$ and $\left|\eta^{*}\right|$ data points were compared with theoretical dynamic mechanical spectrum predicted by the branch-on-branch (BOB) model from the experimental MWDs, as shown in Fig. 9. This model is based on the basic idea of the hierarchical relaxation of branched structures and yields LVE properties by calculating stress relaxation modulus. The predictions assessed using the BOB model for branched polymers matched quite satisfactorily the corresponding experimental data points, as already

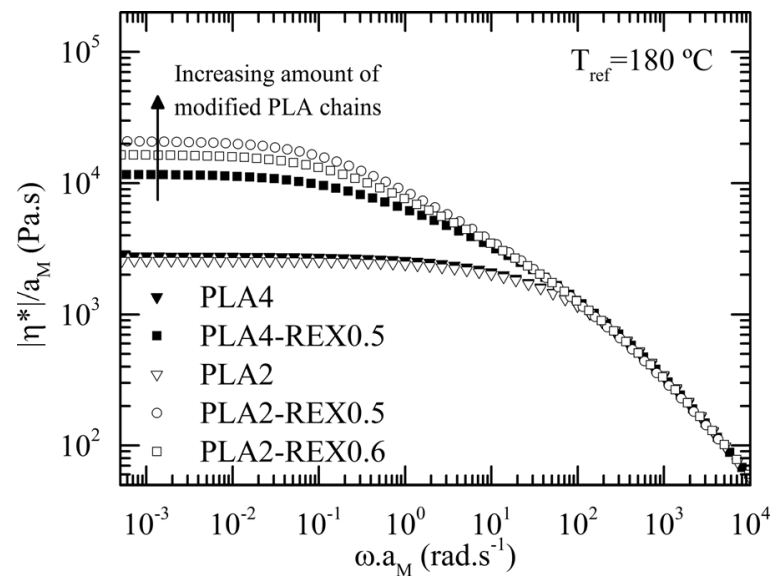

FIG. 8. Shifted complex viscosity for PLA-REX samples together with both linear PLA samples at $180^{\circ} \mathrm{C}$. 
reported by Das et al. (2006) for numerous branched mPE and Liu et al. (2010) for LCB PLA resins. In the present work, the molecular parameters were determined from fitting the experimental data for both linear PLA samples (i.e., PLA4 and PLA2). The density is $1.2 \pm 0.1 \mathrm{~g} \mathrm{~cm}^{-3}$, and the Rouse entanglement time equals $5 \times 10^{-8} \mathrm{~s}$. These parameters are characteristic of PLA and can be used for both PLA and PLA-REX samples. The number of monomers in an entanglement length, $N$, was adjusted as function of the sample in order to adjust properly the plateau modulus region. That is, $N$ equalled 60 for both PLA samples and deceased to 54, 53, and 52 for PLA4-REX0.5, PLA2-REX0.5, and PLA2-REX0.6 samples, respectively.

The LVE predictions for both linear PLA samples fit well the experimental data points. On the contrary, significant discrepancies between the predicted curves and the experimental data points in the low $\omega$ region for all the PLA-REX samples were observed when the entire distribution of MW was assumed to be linear. Experimental points shift to higher values as compared to the simulation traces. Since predictions were entirely based on linear chains, results suggest the presence of macromolecules featuring still
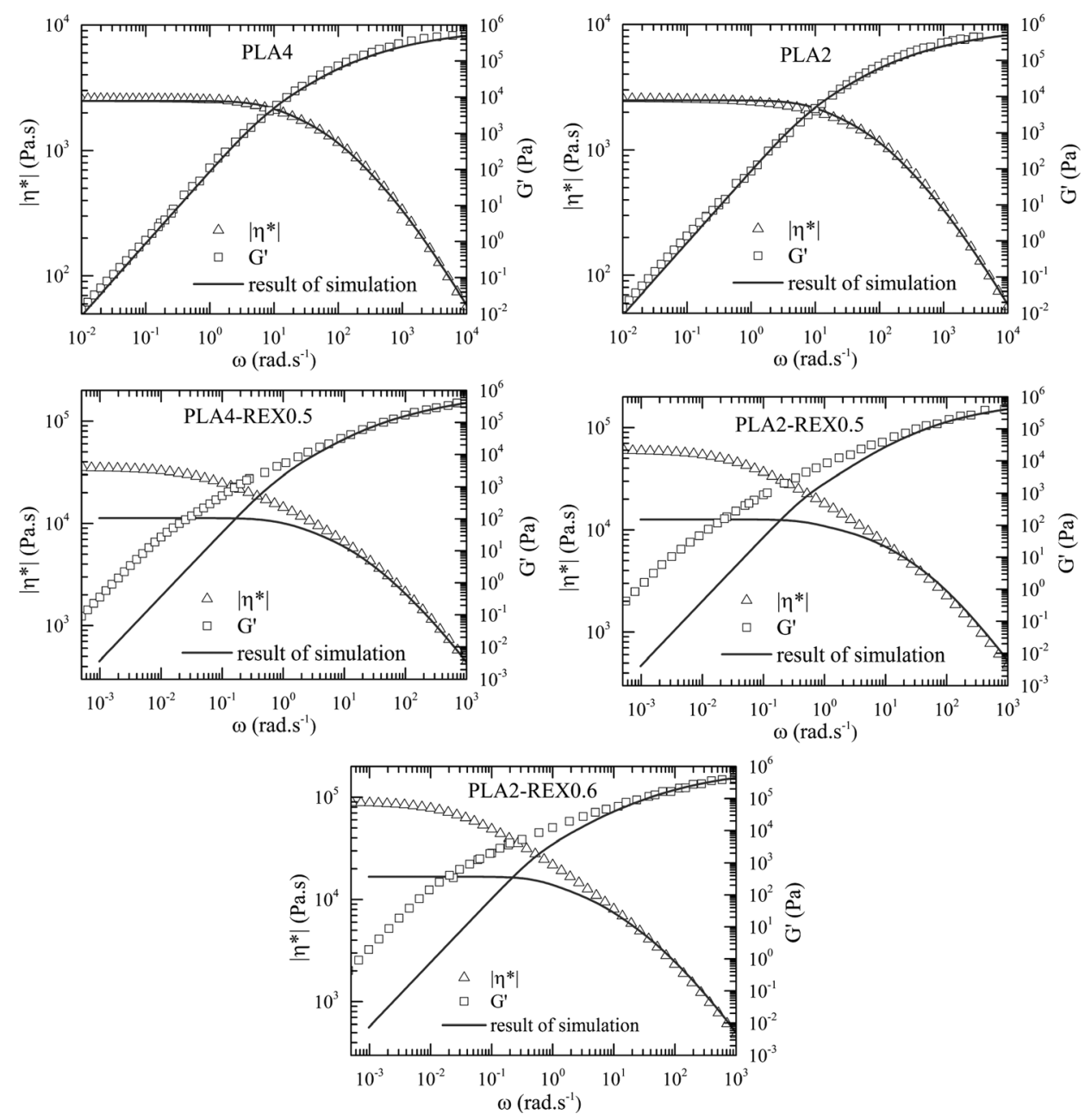

FIG. 9. Experimental (open symbols) and predicted (solid line) LVE properties using the BOB model (assuming that the entire distribution of MW was linear) at $180^{\circ} \mathrm{C}$. 
longer relaxation times and which lead to a completely different spectrum of relaxation times. In other words, this trend implied that PLA-REX samples include some LCB macromolecules.

Due to the higher sensitivity of $\delta$ to molecular structures than the viscosity or storage modulus, the vGP plot is frequently used to get an insight into chain topology. Similarly to $G^{\prime}(\omega)$ and/or $\left|\eta^{*}(\omega)\right|$ master curves, the vGP plots were predicted from the combination of the TTS master curve data referenced at $180^{\circ} \mathrm{C}$ and the conversion of creeprecovery data for thermorheologically simple samples, as shown in Fig. 10. Regarding PLA2-REX0.6 samples, it was obtained from the combination of the viscoelastic measurements at $180^{\circ} \mathrm{C}$ and the conversion of the corresponding creep-recovery data.

The results obtained from the vGP plot appear to agree with the previous results. That is, both PLA samples exhibited the classical behavior of a well entangled linear polymer. The $\delta$ curvature exhibits a limiting plateau value at $90^{\circ}$ for low $\left|G^{*}\right|$ values (viscous behavior) which decreases monotonically up to $0^{\circ}$ for further $\left|G^{*}\right|$ increase (elastic behavior).

In contrast, a completely different scenario was observed for PLA-REX samples. Moving from low to high $\left|G^{*}\right|$ values, $\delta$ decreased from the flow plateau $\left(90^{\circ}\right)$ to its minimum value at lower $\left|G^{*}\right|$ values as compared to PLA samples, but not strictly monotonically. The curves were flattened and $\delta$ values were lowered. Based on the conclusions derived from Figs. 8 and 9, these changes in the vGP plot must be attributed to the presence of topological changes, which hold sparsely LCB, rather than the presence of two fractions of linear chain architectures with different MWs [Trinkle et al. (2002); GarciaFranco et al. (2008)]. This is supported by the fact that PLA-REX samples showed an enhancement of both the $\eta_{0}$ and the transition zone between the terminal and power-law region when the MW effects were removed (cf. Fig. 8) and that the BOB model did not predict accurate viscoelastic properties when the assumed distribution of MW was linear. The gradual shift of $\delta$ toward lower values for both the more reactive PLA type and the increasing SAmfE content match well the above trend (cf. Fig. 8), indicating an increasing content of modified PLA chains. According to Fig. 10, PLA2-REX0.5 showed a higher degree of structural modifications than PLA4-REX0.5 samples for equivalent SAmfE amount, which tendency agrees well with the previous SEC-DRI results (cf. Table IV).

Although topological modifications were developed in PLA-REX samples, the exact chain topology remained unidentified. Based on the detailed classification of LCB polymers by their topology developed by Trinkle et al. (2002), PLA-REX samples did not seem to exhibit a well-defined chain architecture. Due to the statistical nature of the chemical bonding coupled with a possible competition between chain degradation, chain extension, and chain branching, the determination of the branch MW and the branching degree is not trivial. It would be a mixture of several types of nonuniform structures in MW, in architectures and in the number of branch per macromolecules.

Recall that SAmfE agent has a functionality of 12. Thus, several PLA chains were possibly grafted to one SAmfE molecule during processing. In contrast to LCB PEs, PLA chains themselves do not acquire branches. The structure of the resulting modified PLA might be closer to a starlike macromolecule with SAmfE backbone actuating as the branch point. The MW of each arm is supposed to be different due to both the polydispersity of the neat polymer and the effects of the different degradation mechanisms during processing.

The failure of the TTS principle coupled with the usual increase in $E_{a}$ values that would be expected for PLA suspected of containing LCB was not observed for PLA4REX0.5 and PLA2-REX0.5 samples but do not leave out this possibility since these 


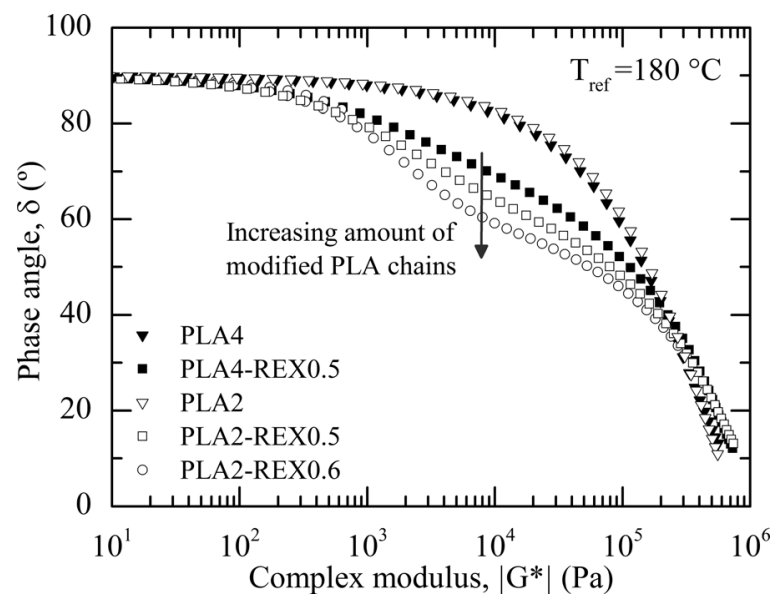

FIG. 10. Effect of the topological modifications on the vGP plot.

materials show an enhancement of both the $\eta_{0}$ and the transition zone between the terminal and power-law region when the MW effects were removed (cf. Fig. 8). Similarly to sparsely LCB HDPE (which is a blend of linear and low levels of branched structures) [Wasserman and Graessley (1996); Vega et al. (1998); Shroff and Mavridis (1999)], this behavior may be attributed to polymers with extremely low fractions of LCB for which the main fraction of remaining linear chains dominates the thermorheological behavior and attenuates the effect of the structural modifications. Regarding PLA2-REX0.6 samples, this behavior changed when the degree of topological change exceeded a critical value. Accordingly, rheological results suggest that the different REX systems could be considered as a mixture between a high fraction of linear chains and a low but increasing amount of nonuniform branched structures, which include sparsely LCB, with both the more reactive PLA type (i.e., PLA2) and the increasing SAmfE content.

PLA4 samples presented slightly higher $M_{w}$ than PLA2 samples as shown in Table IV. Nevertheless, these differences were small and similar melt flow properties at $180^{\circ} \mathrm{C}$ between both samples were also observed in Figs. 5, 6, and 10. These results match those published by Dorgan et al. (2005), who stated that for similar MWs, PLA melt and dilute flow properties remain unaffected by the stereoregularity of the molecular structure. As this work aims to study the effect of the structural modifications on the rheological behavior and on the MWD deduction, from now on only experimental data of PLA4 samples were compared to those of PLA-REX samples, for the sake of clarity.

Based on the changes observed in the $\left|\eta^{*}(\omega)\right|$ behavior with the presence of topological modifications, it is reasonable to expect some modifications in the shape of the deduced MWD spectrum. In Sec. VE, MWDs were determined from melt measurements for PLA4 and PLA-REX samples using the approach of Shaw and Tuminello (1994). Since $\left|\eta^{*}(\omega)\right|$ data must be differentiated twice in Eq. (5), raw data were filtered using values given in Table $\mathrm{V}$ in order to smooth out noises. In addition, all $\left|\eta^{*}(\omega)\right|$ traces were extrapolated at both ends to ensure the prediction of complete MWDs.

\section{E. Effect of the viscosity model on the MWD inferred from melt measurements}

According to the numerical approach developed by Shaw and Tuminello (1994), the MWDs deduced from rheological based data are plotted against the reduced MW $(m)$. 
Scaling $w \log (m)$ toward the MWD is the key point for the robustness of the determination of values of MW and MWD. In order to remove the dependence of the deduced results on $M_{w}$, a relationship relating $M$ to $m$ is suggested [cf. Eq. (19)] from the combination of Eqs. (3b), (6), and (9)

$$
M=m \cdot\left(\eta_{0} / K\right)^{1 / \alpha},
$$

where $\eta_{0}, M$, and $m$ are the zero-shear rate viscosity, the MW, and the reduced MW as defined in Eqs. (11) and (9), respectively. $\alpha$ and $K$ are the exponent and the coefficient of the power-law dependence of $\eta_{0}$ on $M_{w}$ as defined in Eq. (3b), respectively. The use of Eq. (19) with accurate $\alpha$ and $K$ values removes the scaling up and the calibration of the MWD on chromatographic data.

For the purpose to investigate how structural modifications affect the $\eta_{0}-M_{w}$ correlation, $\eta_{0}$ values at $180^{\circ} \mathrm{C}$ as a function of the relative $M_{w}$, as determined using SEC-DRI (cf. Table IV), are plotted in Fig. 11. Experimental data points from Dorgan et al. (2005) were also added in order to enlarge the span of MWs considered. The dashed line represents the relation found for linear HMW PLA with an exponent $\alpha$ of $3.4 \pm 0.1$ and a coefficient $K$ of $5.5 \times 10^{-15}$ [Dorgan et al. (2005)]. It is apparent that the data corresponding to PLA4 samples matched well the previous relation.

However, a clear deviation of $\eta_{0}\left(M_{w}\right)$ from $\eta_{0} \approx M_{w}^{3.4}$ toward higher viscosity values was found for PLA-REX samples. Since the power-law dependence of $\eta_{0}$ on $M_{w}$ is one of the relationships which remains independent of sample polydispersity [Gabriel and Munstedt (2002); Auhl et al. (2004); Gabriel and Munstedt (2002); Auhl et al. (2004)], the minor variations in the breath of the MWD between PLA and PLA-REX samples (cf. Table IV) cannot account for this deviation from the dashed line.

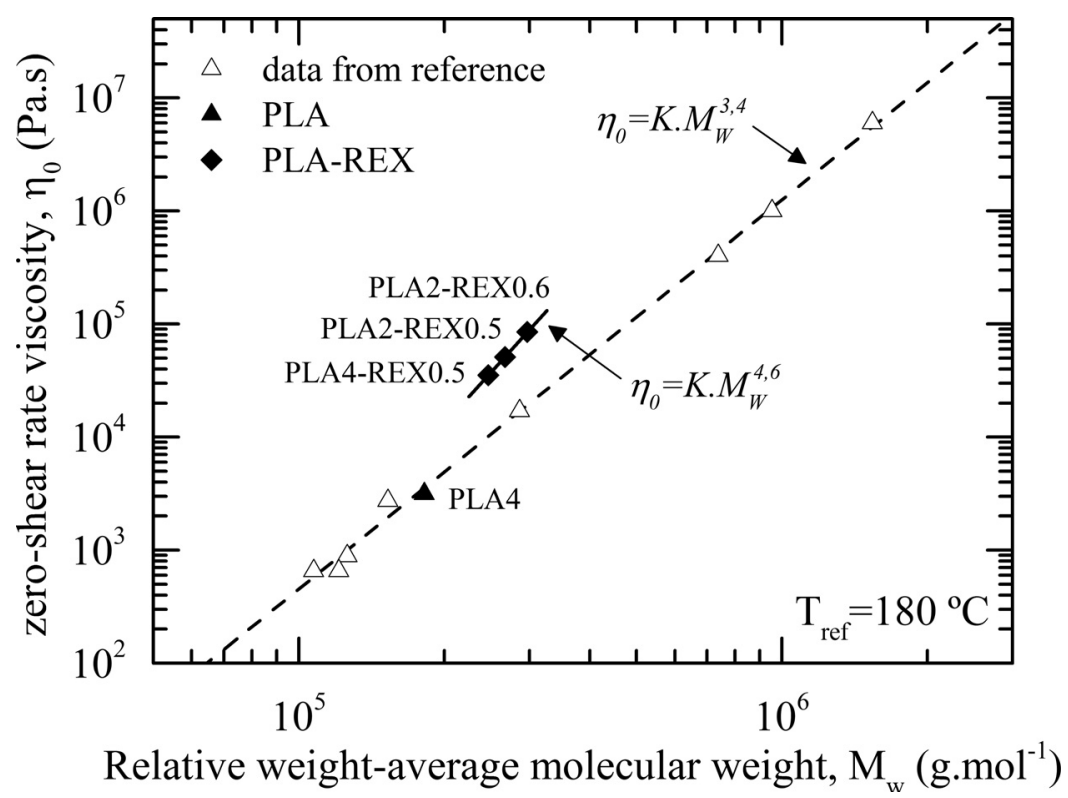

FIG. 11. Plot of the zero-shear rate viscosity at $180^{\circ} \mathrm{C}$ with the relative weight-average $\mathrm{MW}$ as determined using SEC-DRI. The dashed line indicates the correlation between $\eta_{0}$ and $M_{w}$ established by Dorgan et al. (2005) with the corresponding data points (open triangles). 
For the purpose to scale $w \log (m)$ toward the absolute MWD without resorting of chromatographic data [cf. Eq. (19)] for PLA-REX samples, a power-law dependence could be considered as reliable in order to plot $\eta_{0}$ with $M_{w}$. This is due to the narrow span of MWs covered by REX samples $\left(246-292 \mathrm{~kg} \mathrm{~mol}^{-1}\right)$. Over the MW covered, an exponent $\alpha$ of $4.6 \pm 0.1$ and a coefficient $K$ of $1.3 \times 10^{-21}$ were measured for PLA-REX samples. Dorgan et al. (1999) reported an even stronger scaling factor regarding 4 and 6 arms starlike PLA polymers.

On this basis, the viscosity MWDs deduced from melt measurements were calculated and results were compared to the SEC-DRI data as shown in Fig. 12. $m$ was scaled up with MW using Eq. (19) and the respective results obtained from Fig. 11. The reliability of the procedure for determining MWD from the inversion technique is central to the technique proposed here for the determination of the MWD of LCB polymers. MW value averages with respect to the fitting model considered are compiled in Table VI. The error percentages calculated arise from the difference between the deduced values and the SEC-DRI values (cf. Table IV).

Regarding PLA4 samples, viscosity MWDs fit well to the experimental SEC-DRI data as shown in Fig. 12. A good agreement was observed on the location of the main peak and on the width of the MWD. Discrepancies in the HMW tail between the inversion technique predictions and the SEC data possibly is a consequence of the assumed lognormal distribution for the calculated MWDs. Similar quantitative accuracy between both inferred and SEC MWDs has been reported by Wood-Adams and Dealy (2000) for linear PEs. The principal point here is that the $\mathrm{CY}$ and $\mathrm{HN}$ models appear to provide equivalent molecular information for a linear polymer with a PDI of about two.

The robustness of the HN model to capture the fundamental molecular information resides in its mathematical structure. Gross (1953) stated that the shape of the relaxation spectrum $(H(\lambda))$ is accurately described by an analytical expression derived from Eq. (13). If the Cox-Merz rule holds, a direct correlation may be established between the CY parameters and the $H(\lambda)$ parameters. Thus, the phenomenological model initially developed to describe nonlinear steady-flow properties possibly contains similar molecular information as the HN model.

The two different relaxation regimes observed in the trace of $\left|\eta^{*}(\omega)\right|$ between the Newtonian and shear-thinning regimes for PLA-REX samples are here reflected in terms of bimodal distribution of MW. Regardless of the fitting model considered, the MW average and PDI values agreed within 10\% between melt and SEC-DRI measurements. However, the peak positions and the shape of the MWDs between both measurements did not match. The position of the second peak (marked as peak II) was fairly constant between all measurements. Nevertheless, the peak MW $\left(M_{p}\right)$ of the first peak (marked as peak I), which is inferred by melt measurements, shifted to lower MWs as compared to SEC data. Similar differences have been reported in Wood-Adams and Dealy (2000) for LCB PEs.

Figure 13 reports the $M_{p}$ values of the first peak obtained from SEC-DRI and melt measurements, respectively. While SEC data depict a constant increase in $M_{p}$ with the amount of topological modifications, $M_{p}$ values estimated from melt measurements were roughly centerd in similar MW values between PLA and PLA-REX samples.

Since $\left|\eta^{*}(\omega)\right|$ is related to the MWD using the mixing rule developed by Malkin and Teishev (1991) [cf. Eq. (4)], the bimodal viscosity MWDs should be interpreted as the summation of two distinct distributions of MW. Both of them are correlated with each separated relaxation process exhibited in the $\left|\eta^{*}(\omega)\right|$ trace. Peak I was related to the linear chain fraction in all spectra due to the linear architecture of PLA4 samples. This signal is governed by the second transition region in $\left|\eta^{*}(\omega)\right|[i=2$ in Eqs. (12) and (15)] as the 


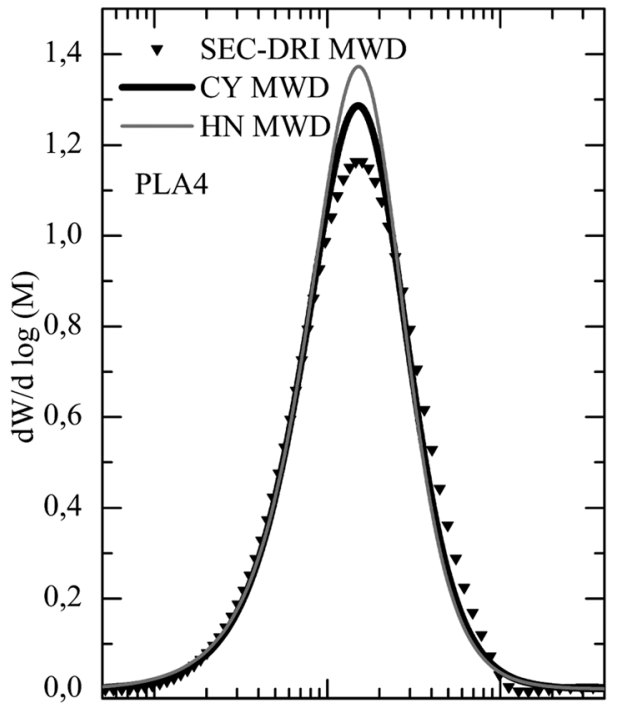

$10^{4} \quad 10^{5} \quad 10^{6}$

Molecular weight, $\mathrm{M}\left(\mathrm{g} \cdot \mathrm{mol}^{-1}\right)$

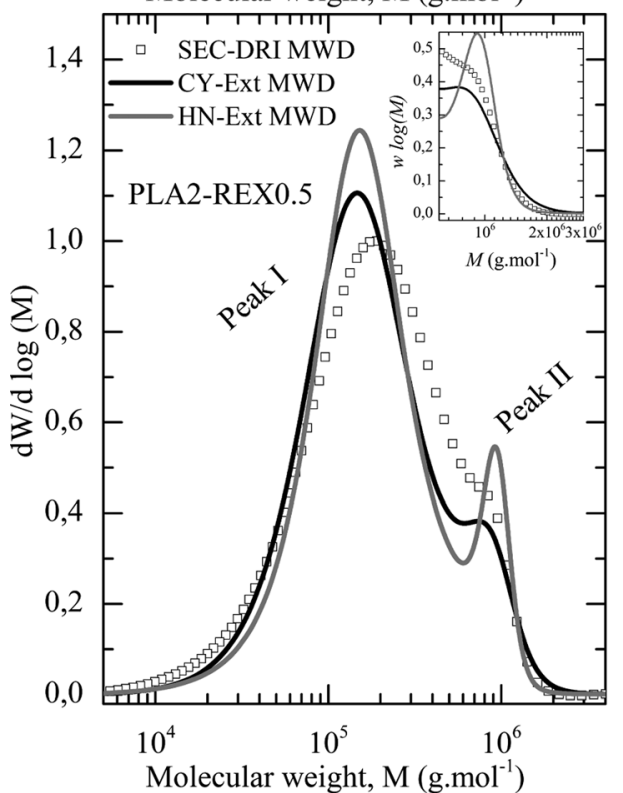

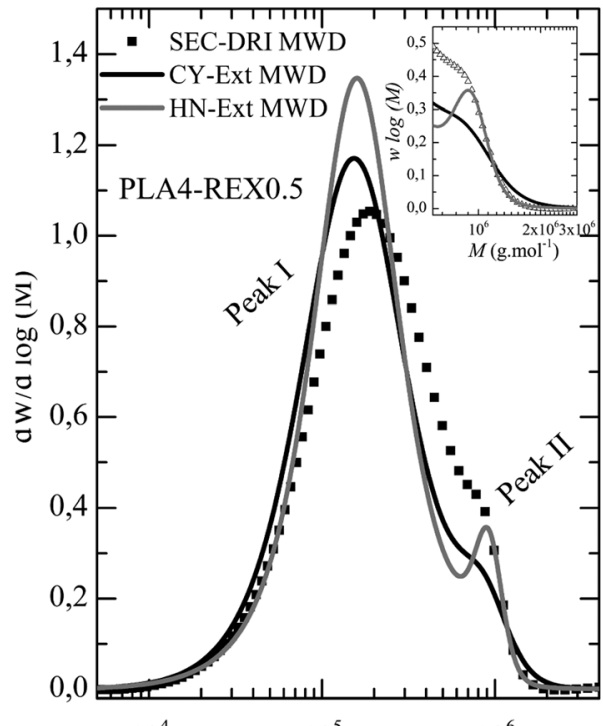

$10^{4}$

Molecular weight, $\mathrm{M}\left(\mathrm{g} \mathrm{mol}^{-1}\right)$

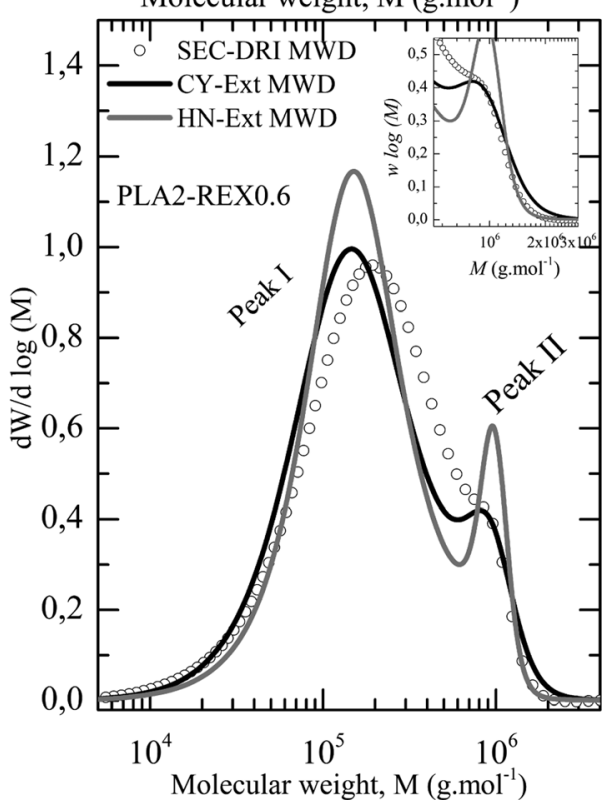

FIG. 12. Effect of the viscosity fitting model on the MWD deduced from melt measurements. Insets show a detailed view of the HMW tails regarding bimodal distributions.

MW is inversely related to $\omega$ by combining Eqs. (6) and (9). It remained centerd in roughly similar MW values due to a possible similar time dependence of the relaxation processes governing the linear population in PLA and PLA-REX samples. The second peak at HMW is ruled by the intermediate relaxation regime in the $\left|\eta^{*}(\omega)\right|$ trace $[i=1$ in Eqs. (12) and (15)], resulting from the longer relaxation times induced by the topological changes.

Despite the fact that the second peak position appeared to be located in good agreement between all measurements, its resolution was greatly affected by the viscosity 
TABLE VI. Effect of the viscosity fitting model on the MW value averages.

\begin{tabular}{lccccc}
\hline \hline Sample & Fitting model & $M_{n}\left(\mathrm{~kg} \mathrm{~mol}^{-1}\right)$ & $M_{w}\left(\mathrm{~kg} \mathrm{~mol}^{-1}\right)$ & $M_{z}\left(\mathrm{~kg} \mathrm{~mol}^{-1}\right)$ & $M_{w} / M_{n}$ \\
\hline PLA4 & CY & 97 & 185 & 357 & 1.90 \\
& Error $(\%)^{\mathrm{a}}$ & 5.5 & 0.1 & 8.2 & 5.9 \\
& HN & 93 & 181 & 350 & 1.93 \\
& Error $(\%)^{\mathrm{a}}$ & 2.0 & 2.6 & 6.2 & 4.7 \\
PLA4-REX0.5 & CY-Ext & 110 & 245 & 530 & 2.24 \\
& Error $(\%)^{\mathrm{a}}$ & 8.33 & 0.09 & 10.48 & 8.23 \\
& HN-Ext & 118 & 245 & 493 & 2.08 \\
& Error $(\%)^{\mathrm{a}}$ & 0.55 & 0.1 & 3.65 & 0.44 \\
PLA2-REX0.5 & CY-Ext & 114 & 272 & 595 & 2.39 \\
& Error $(\%)^{\mathrm{a}}$ & 7.2 & 0.1 & 8.4 & 7.0 \\
& HN-Ext & 115 & 278 & 572 & 2.28 \\
& Error $(\%)^{\mathrm{a}}$ & 7.8 & 2.1 & 4.8 & 11 \\
PLA2-REX0.6 & CY-Ext & 107 & 292 & 666 & 2.73 \\
& Error $(\%)^{\mathrm{a}}$ & 11.8 & 0.1 & 9.3 & 10.5 \\
& HN-Ext & 121 & 294 & 618 & 2.44 \\
& Error $(\%)^{\mathrm{a}}$ & 0.8 & 0.6 & 2.2 & 0.2 \\
\hline \hline
\end{tabular}

${ }^{\text {a }}$ The error percentages arise from the difference between the deduced values from melt measurements and the SEC-DRI values reported in Table IV.

model used to fit $\left|\eta^{*}(\omega)\right|$ data. The bimodality appeared to be better resolved when the HN-Ext model was used. This enhanced resolution could be a consequence of the incorporation of the complex time dependence of the viscoelastic response into the characterization.

As the Cox-Merz assumption does not hold for LCB polymers, a direct correlation between the CY-Ext parameters and the $H(\lambda)$ parameters cannot be established. A loss of subtle information about molecular and relaxation processes possibly occur when the

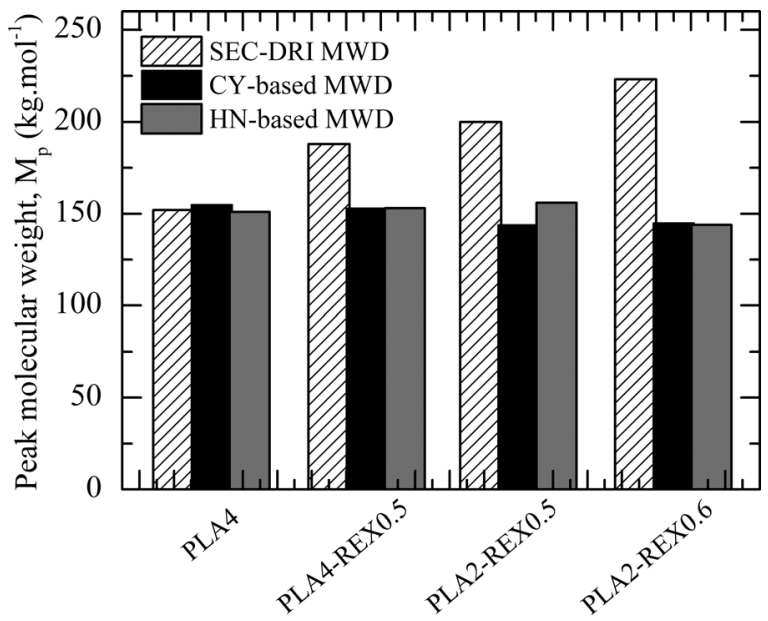

FIG. 13. Comparison of $M_{p}$ for the first peak deduced from SEC-DRI and melt measurements. CY-based MWD: MWD inferred from viscosity functions fitted using the CY model for PLA4 samples and using the CYExt model for PLA-REX samples. HN-based MWD: MWD inferred from viscosity functions fitted using the HN model for PLA4 samples and using the HN-ext model for PLA-REX samples. 
CY-Ext model is used to fit $\left|\eta^{*}(\omega)\right|$ data. The flattening of the HMW peak might be a consequence of this fitting issue.

Figure 14 depicts the effects of the increasing amount of topological modifications on the viscosity MWDs. MWDs inferred from the inversion of the HN-Ext viscosity model were preferred to show differences between PLA-REX samples. This was due to the larger quantification observed between the molecular information related to the linear and modified chain fractions, respectively.

During processing, several PLA chains are possible grafted to one SAmfE molecule. As shown in Figs. 8 and 10, the content of modified PLA chains increased at the expense of the linear chain fraction for both the more reactive PLA type (i.e., PLA-2) and the increasing SAmfE content. In Fig. 14, the evolution of the height of both peaks suggests that the extent of the relaxation processes related to the modified chain fraction grows at the expense of those related to the linear chain fraction. The observed trend could be assumed as characteristic of an increasing content of topological changes.

\section{F. Quantitative estimation of the content of modified chains}

The degree of branching for polyolefins is usually reported as the number of LCB's per 10000 carbon atoms. However, this definition is inapplicable to characterize PLAREX samples. This is due to the probable mixture of various sparsely nonuniform branched structures generated through REX. Numerous intents to evaluate the structural modifications have been performed using SEC-multiple detectors (as detailed in Sec. V A) and ${ }^{1} \mathrm{H}$ NMR (as detailed below) experiments. Nevertheless, unreliable results were obtained due to an architecturally modified chain content possibly below the detection limit of these techniques and to the nonuniformity of the different branched macromolecules. Based on the above rheological data, viscoelastic measurements in the melted state appeared to be an effective method to detect such low amounts of nonuniform topological changes. Thus, this study aims to quantify the content of modified PLA chains solely based on melt measurements.

In Fig. 14, the observed pattern could be used to estimate a content of modified chains simply from knowledge of viscosity MWD alone. The evolution of the peak corresponding to the modified chain topology (peak II) was followed using a deconvolution analysis. All MWD spectra were analyzed using log-normal functions [cf. Eq. (20)] with the condition of finding two peaks centered at the same maxima as exhibited by MWD traces. The partial area below the peak II was related to a quantitative amount of modified PLA chains. Results are given in Table VII

$$
\frac{d W}{d \log (M)}=\frac{a_{0}}{\sqrt{2 \pi} * a_{1} * a_{2} * \sqrt{\exp \left(\mathrm{a}_{2}^{2}\right)}} * \exp \left(-\frac{1}{2}\left(\frac{\frac{\ln M}{a_{1}}}{a_{2}}\right)^{2}\right),
$$

where $a_{0}, a_{1}$, and $a_{2}$ are numerical parameters related to the area, the center, and the width of the log-normal function, respectively.

By examining the obtained data, an apparent increase in the amount of modified chains was observed for both the more reactive PLA type (i.e., PLA2) and the increasing SAmfE content. However, calculated data have to be compared with other characterization techniques in order to verify the reliability of the results, due to the novelty of this technique. 


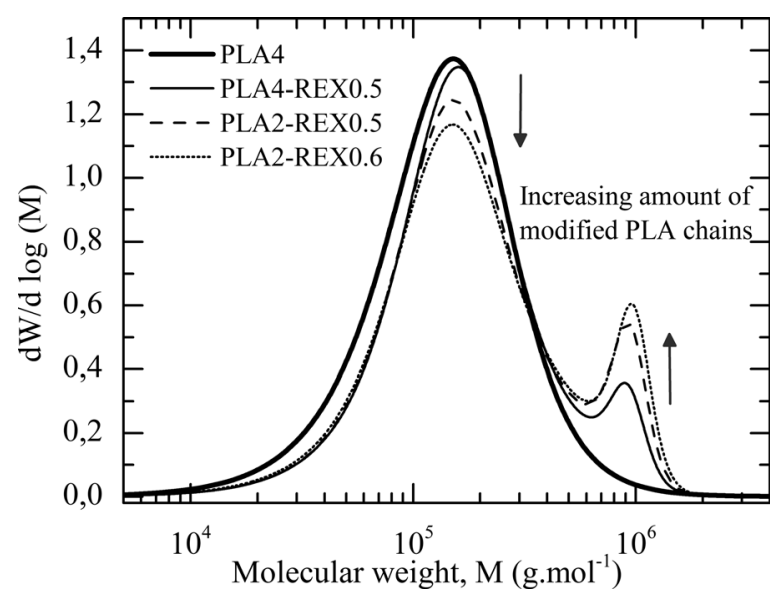

FIG. 14. Effect of an increasing content of topological modifications on the MWD inferred from melt measurements.

In the present work, the contrast between the eluted linear and modified chain fractions was enhanced using SEC-DRI measurements. As previously discussed, the shoulder in the HMW tail of SEC data may indicate an increase in the population of HMW linear chains and/or the presence of branching. In order to quantify a relative amount of modified chains from a chromatographic technique, a similar peak deconvolution technique as described above was used. The partial area below the shoulder was related to a quantitative amount of modified PLA chains as summarized in Table VII.

A preliminary trial was performed to characterize the molecular structure using ${ }^{1} \mathrm{H}$ NMR experiments. ${ }^{1} \mathrm{H}$ NMR spectra of both PLA4 and PLA-REX samples with the highest amount of structural modifications (i.e., PLA2-REX0.6) are shown in Fig. 15 together with peak assignments.

In both spectra, the most intense signals at 1.8 and $5.2 \mathrm{ppm}$ correspond to methyl $\left(-\mathrm{CH}_{3}\right)$ and methine $(-\mathrm{CH})$ protons at the (c) and (b) positions, respectively, which belong to the main PLA chain. The resonance peaks at 1.04 and $4.4 \mathrm{ppm}$ are thought to belong to the $-\mathrm{CH}_{3}$ and $-\mathrm{CH}$ protons next to the terminal $-\mathrm{OH}$ groups (i.e., (d) and (a) positions, respectively). Note that the sharp peak at $7.29 \mathrm{ppm}$ is due to the deuterated chloroform $\mathrm{CDCl}_{3}$ [Espartero et al. (1996); Meng et al. (2012)].

The broad peak, which overlaps the signal of the solvent (indicated by an arrow) for PLA2-REX0.6 samples, probably belongs to the protons in the aromatic rings of SAmfE molecules. While the coupling reactions between PLA and a multiepoxy reactive agent have been identified in Meng et al. (2012), both spectra remained similar in the present

TABLE VII. Quantitative estimation of the amount of modified PLA chains.

\begin{tabular}{lccc}
\hline \hline & \multicolumn{3}{c}{ Amount of modified PLA chains (\%) } \\
\cline { 2 - 4 } Sample & Melt measurements $^{\mathrm{a}}$ & SEC-DRI $^{\mathrm{b}}$ & NMR \\
\hline PLA4-REX0.5 & 22 & 22 & 19 \\
PLA2-REX0.5 & 24 & 25 & 24 \\
PLA2-REX0.6 & 32 & 29 & 29 \\
\hline \hline
\end{tabular}

${ }^{\text {a }}$ Partial area below the second peak in the MWD inferred from melt measurements.

${ }^{\mathrm{b}}$ Partial area below the shoulder in the HMW tail of the SEC-DRI data. 
work. Similar observations were highlighted for PLA4-REX0.5 and PLA2-REX0.5 samples; suggesting the absence of branched structures. It must be kept in mind that NMR cannot detect sparsely and/or nonuniform branched macromolecules and cannot distinguish LCB to the main polymer backbone. This is because both give rise to the same spectral pattern irrespective of the chain length. However, the rheologically relevant branches are those long enough to entangle, as it was shown in Sec. VD.

On the other hand, ${ }^{1} \mathrm{H}$ NMR data permitted to estimate an increase in the number of terminal groups between PLA and PLA-REX samples. Similar magnetic resonance properties between both the linear PLA chains and the PLA chains grafted to SAmfE molecules were assumed in this approach, as schematically illustrated in Fig. 16.

The relative number of resonance protons in a polymeric chain is related to the integration of the corresponding resonance peak intensities (I). For linear PLA, the analysis of the integration ratio between the (a) and (b) peaks leads to an absolute value of the degree of polymerization $\left(D P_{n}=I_{b} / I_{a}\right)$. This procedure solely remains valid for linear PLA chains as they bear only one hydroxyl end group close to the (a) position. For PLAREX samples, the number of hydroxyl groups per macromolecule, close to the (a) position, possibly increased because branched polymers are characterized by the presence of branch points and the presence of more than two end groups. Therefore, the $D P_{n}$ of

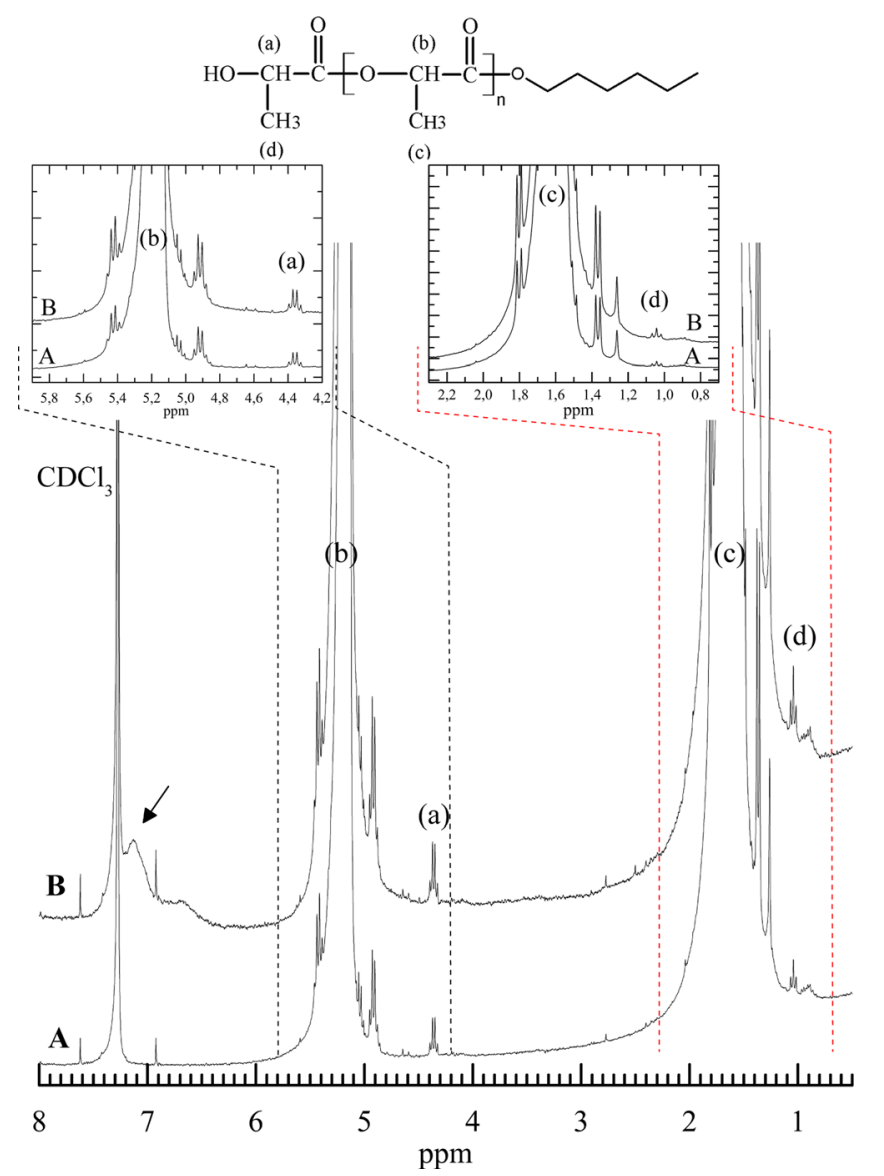

FIG. 15. ${ }^{1} \mathrm{H}$ NMR spectra of (A) PLA4 and (B) PLA2-REX0.6 with peak assignments. The arrow indicates the signal which probably belongs to the protons in the aromatic rings of the SAmfE molecules. 
PLA-REX sample $\left(\left(D P_{n}\right)_{P L A-R E X}\right)$ is possibly underestimated as compared to its linear homologue $\left(\left(D P_{n}\right)_{P L A}\right)$. For a given MW, $\left(D P_{n}\right)_{P L A}$ was related to $\left(D P_{n}\right)_{P L A-R E X}$ using a correlation factor $K$, which was defined as an average of terminal groups, as shown below

$$
\left(D P_{n}\right)_{P L A}=K *\left(D P_{n}\right)_{P L A-R E X} .
$$

However, the increasing MW values upon SAmfE addition suggest that Eq. (21) required MW correction using SEC-DRI experiments. Thus, the $D P_{n}$ ratio between PLA-REX and PLA samples measured by NMR was related to those obtained from the ratio of molar masses measured by SEC as follows:

$$
\left[\frac{\left(D P_{n}\right)_{P L A-R E X}}{\left(D P_{n}\right)_{P L A}}\right]_{N M R} * K=\left[\frac{\left(D P_{n}\right)_{P L A-R E X}}{\left(D P_{n}\right)_{P L A}}\right]_{S E C},
$$

where $\left[\left(D P_{n}\right)\right]_{R M N}=I_{b} / I_{a} . I_{a}$ and $I_{b}$ are the integral areas of the methine proton at the (a) and (b) position, respectively. $\left[\left(D P_{n}\right)\right]_{S E C}=M_{n} / M_{0} . M_{n}$ is the relative number-average MW and $M_{o}$ the MW of the repeat unit. In the current study, the integration ratio between both resonance peaks was normalized with respect to one methine proton at the (a) position $\left(I_{a}=1\right)$ for all samples.

An increasing number of terminal groups was related to the amount of modified PLA chains $(\Delta K)$ which can be calculated from Eq. (23). Numerical values are summarized in Table VII

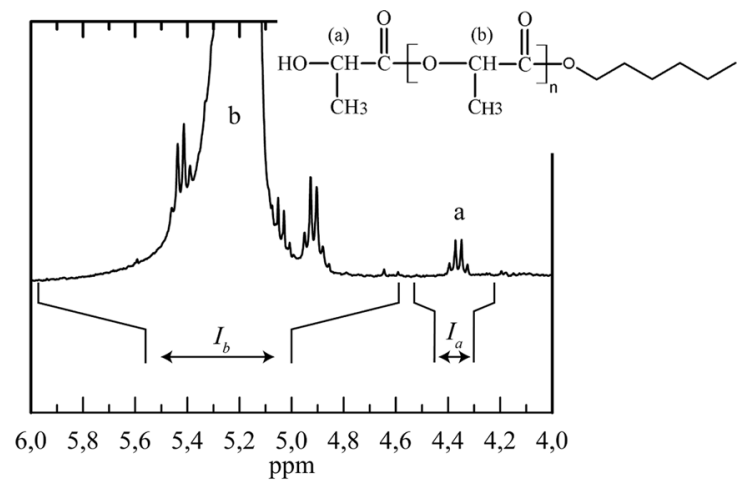

PLA

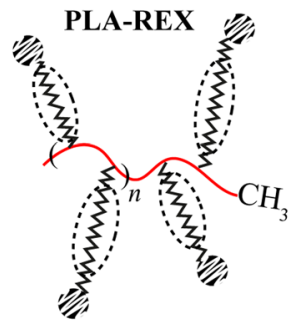

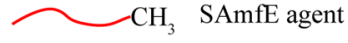

........ PLA chain segments with similar NMR properties: $I_{b}$
NMR properties of methine groups close to the terminal -OH group: $I_{a}$

FIG. 16. Illustration of the integral analysis for ${ }^{1} \mathrm{H}$ NMR measurements. 


$$
\Delta K(\%)=\left(\frac{\left(I_{b}\right)_{P L A}}{\left(I_{b}\right)_{P L A-R E X}} * \frac{M_{n P L A-R E X}}{M_{n P L A}}-1\right) * 100
$$

where $\left(I_{b}\right)_{P L A}$ and $\left(I_{b}\right)_{P L A-R E X}$ are the integral areas of the methine proton at the (b) position of the PLA and PLA-REX samples, respectively. $M_{n}$ PLA-REX and $M_{n}$ PLA are the relative number-average MWs of PLA-REX and of the PLA sample type as determined using SEC-DRI (cf. Table IV). It should be noted that the repeatability of the measurements was in the order of $\pm 10 \%$.

For each sample, all measurements were largely similar as shown in Table VII. Consequently, the technique developed from solely melt measurement can be considered as reliable to quantitatively estimate the amount of modified chains. A quantitatively low but increasing amount of modified chains was determined for both the more reactive PLA type (i.e., PLA2) and the increasing SAmfE content.

\section{CONCLUSIONS}

The incorporation of a multifunctionalized epoxy agent into two commercial PLA extrusion grades was performed through REX. The application of the TMW superposition principle provided the sensitivity to distinguish the effects induced by the increase in MW from that of the topological modifications. According to the processing conditions, rheological properties highlighted the presence of low levels of nonuniform branched structures which held sparsely LCB. Similarly to sparsely LCB mPE, the usual enhancement of the flow activation energy and the loss of the thermorheologically simple behavior for samples containing LCB were not observed for PLA-REX samples exhibiting an amount of modified chains up to approximately $24 \%$.

The inversion technique developed by Shaw and Tuminello (1994) was successfully applied to LCB polymers featuring bimodal distribution of MWs with a PDI of about two. Regardless of the viscosity fitting model used to extrapolate $\left|\eta^{*}(\omega)\right|$ data, predicted MW average and PDI values agreed with SEC-DRI results within $10 \%$. A larger quantification between the molecular information related to the linear and modified chain fractions was observed with the introduction of the complex time dependence into the MWD prediction.

Based on the inferred MWD spectra, a method was described to quantitatively estimate the amount of modified PLA chains. The described technique allows a rapid and consistent approach to quantify low levels of structural modification in PLA resins which may be important for commercial systems.

\section{ACKNOWLEDGMENTS}

The authors would like to acknowledge the financial support from the Spanish Ministry of Economy and Competitiveness through the Project MAT2013-40730-P. BASF is thanked for kindly supplying the reactive agent and Irganox 1010.

\section{References}

Al-Itry, R., K. Lamnawar, and A. Maazouz, "Reactive extrusion of PLA, PBAT with a multi-functional epoxide: Physico-chemical and rheological properties," Eur. Polym. J. 58, 90-102 (2014). 
Auhl, D., J. Stange, H. Munstedt, B. Krause, D. Voigt, A. Lederer, U. Lappan, and K. Lunkwitz, "Long-chain branched polypropylenes by electron beam irradiation and their rheological properties," Macromolecules 37, 9465-9472 (2004).

Bersted, B. H., and J. D. Slee, "Relationship between steady-state shear melt viscosity and molecular-weight distribution in polystyrene," J. Appl. Polym. Sci. 21, 2631-2644 (1977).

Bigg, D. M., "Polylactide copolymers: Effect of copolymer ratio and end capping on their properties," Adv. Polym. Technol. 24, 69-82 (2005).

Bikiaris, D. N., and G. P. Karayannidis, "Chain extension of polyesters PET and PBT with two new diimidodiepoxides. 2.,” J. Polym. Sci., Part A: Polym. Chem. 34, 1337-1342 (1996).

Cailloux, J., O. O. Santana, E. Franco-Urquiza, J. J. Bou, F. Carrasco, J. Gamez-Perez, and M. L. Maspoch, "Sheets of branched poly(lactic acid) obtained by one step reactive extrusion calendering process: Melt rheology analysis," eXPRESS Polym. Lett. 7, 304-318 (2013).

Carlson, D., P. Dubois, L. Nie, and R. Narayan, "Free radical branching of polylactide by reactive extrusion," Polym. Eng. Sci. 38, 311-321 (1998).

Carreau, P. J., S. H. Tabatabaei, and A. Ajji, "Rheological long-chain properties of blends of linear and branched polypropylenes," Polym. Eng. Sci. 50, 191-199 (2010).

Chen, X. Y., and Y. M. Zhang, "Determining molecular weight scale and molecular weight distribution of ethylene-tetrafluoroethylene alternating copolymer via a rheological technique," J. Appl. Polym. Sci. 125, 2442-2448 (2012).

Cocchini, F., and M. R. Nobile, "Constrained inversion of rheological data to molecular weight distribution for polymer melts,” Rheol. Acta 42, 232-242 (2003).

Corre, Y. M., J. Duchet, J. Reignier, and A. Maazouz, "Melt strengthening of poly (lactic acid) through reactive extrusion with epoxy-functionalized chains,” Rheol. Acta 50, 613-629 (2011).

Crosby, B. J., M. Mangnus, W. de Groot, R. Daniels, and T. C. B. McLeish, "Characterization of long chain branching: Dilution rheology of industrial polyethylenes," J. Rheol. 46, 401-426 (2002).

Das, C., N. J. Inkson, D. J. Read, M. A. Kelmanson, and T. C. B. McLeish, "Computational linear rheology of general branch-on-branch polymers," J. Rheol. 50, 207-235 (2006).

Dealy, J., H. E. Park, and H. Munstedt, "Influence of long-chain branching on time-pressure and timetemperature shift factors for polystyrene and polyethylene," Rheol. Acta 46, 153-159 (2006).

Dealy, J. M., and R. G. Larson, "Determination of molecular weight distribution using rheology," in Structure and Rheology of Molten Polymers: From Structure to Flow Behavior and Back Again, edited by J. M. Dealy and R. G. Larson (Hanser Publisher, Munich, 2006a).

Dealy, J. M., and R. G. Larson, "Linear viscoelasticity-behavior of molten polymers," in Structure and Rheology of Molten Polymers: From Structure to Flow Behavior and Back Again, edited by J. M. Dealy and R. G. Larson (Hanser Publisher, Munich, 2006b).

Dealy, J. M., and R. G. Larson, "Linear viscoelasticity-fundamentals," in Structure and Rheology of Molten Polymers: From Structure to Flow Behavior and Back Again, edited by J. M. Dealy and R. G. Larson (Hanser Publisher, Munich, 2006c).

Di, Y. W., S. Iannace, E. Di Maio, and L. Nicolais, "Reactively modified poly(lactic acid): Properties and foam processing,” Macromol. Mater. Eng. 290, 1083-1090 (2005).

Doerpinghaus, P. J., and D. G. Baird, "Separating the effects of sparse long-chain branching on rheology from those due to molecular weight in polyethylenes," J. Rheol. 47, 717-736 (2003).

Dorgan, J. R., J. Janzen, M. P. Clayton, S. B. Hait, and D. M. Knauss, "Melt rheology of variable L-content poly(lactic acid),” J. Rheol. 49, 607-619 (2005).

Dorgan, J. R., J. S. Williams, and D. N. Lewis, "Melt rheology of poly(lactic acid): Entanglement and chain architecture effects,” J. Rheol. 43, 1141-1155 (1999).

Elster, C., and J. Honerkamp, "The role of the error model in the determination of the relaxation time spectrum," J. Rheol. 36, 911-927 (1992).

Espartero, J. L., I. Rashkov, S. M. Li, N. Manolova, and M. Vert, "NMR analysis of low molecular weight poly(lactic acid)s," Macromolecules 29, 3535-3539 (1996).

Ferri, D., and P. Lomellini, "Melt rheology of randomly branched polystyrenes," J. Rheol. 43, 1355-1372 (1999). 
Ferry, J. D., Viscoelastic Properties of Polymers, 3rd ed. (Wiley, NY, 1980).

Fleissner, M., "Characterization of polymer molecular mass-distribution from rheological measurements," Makromol. Chem. Macromol. Symp. 61, 324-341 (1992).

Gabriel, C., and H. Munstedt, "Creep recovery behavior of metallocene linear low-density polyethylenes," Rheol. Acta 38, 393-403 (1999).

Gabriel, C., and H. Munstedt, "Influence of long-chain branches in polyethylenes on linear viscoelastic flow properties in shear," Rheol. Acta 41, 232-244 (2002).

Gabriel, C., E. Kokko, B. Lofgren, J. Seppala, and H. Munstedt, “Analytical and rheological characterization of long-chain branched metallocene-catalyzed ethylene homopolymers," Polymer 43, 6383-6390 (2002).

Garcia-Franco, C. A., and D. W. Mead, "Rheological and molecular characterization of linear backbone flexible polymers with the Cole-Cole model relaxation spectrum," Rheol. Acta 38, 34-47 (1999).

Garcia-Franco, C. A., D. J. Lohse, C. G. Robertson, and O. Georjon, "Relative quantification of long chain branching in essentially linear polyethylenes,” Eur. Polym. J. 44, 376-391 (2008).

Gotsis, A. D., B. L. F. Zeevenhoven, and C. J. Tsenoglou, "Effect of long branches on the rheology of polypropylene," J. Rheol. 48, 895-914 (2004).

Gross, B., Mathematical Structure of the Theories of Viscoelasticity (Hermann, Paris, 1953).

Havriliak, S., and S. Negami, "A complex plane representation of dielectric and mechanical relaxation processes in some polymers," Polymer 8, 161-210 (1967).

Honerkamp, J., and J. Weese, "A nonlinear regularization method for the calculation of relaxation spectra," Rheol. Acta 32, 65-73 (1993).

Janzen, J., and R. H. Colby, “Diagnosing long-chain branching in polyethylenes,” J. Mol. Struct. 485, 569-584 (1999).

Joncryl ADR-4300, Technical Data Sheets, 2006, electronic file accessible at http://www2.basf.us

Kasehagen, L. J., C. W. Macosko, D. Trowbridge, and F. Magnus, "Rheology of long-chain randomly branched polybutadiene," J. Rheol. 40, 689-709 (1996).

Kratochvil, P., "Characterization of branched polymers," Macromol. Symp. 152, 279-287 (2000).

Lagendijk, R. P., A. H. Hogt, A. Buijtenhuijs, and A. D. Gotsis, "Peroxydicarbonate modification of polypropylene and extensional flow properties," Polymer 42, 10035-10043 (2001).

Lai, S., T. A. Plumley, T. I. Butler, G. W. Knight, and C. I. Kao, "Dow rheology index (DRI) for insite technology polyolefins (ITP): Unique structure-processing relationships,” Annu. Tech. Conf. Soc. Plast. Eng. 40, 1814-1815 (1994).

Lehermeier, H. J., and J. R. Dorgan, "Melt rheology of poly(lactic acid): Consequences of blending chain architectures,” Polym. Eng. Sci. 41, 2172-2184 (2001).

Liu, J. Y., L. J. Lou, W. Yu, R. G. Liao, R. M. Li, and C. X. Zhou, "Long chain branching polylactide: Structures and properties," Polymer 51, 5186-5197 (2010).

Liu, W., D. G. Ray III, and P. L. Rinaldi, "Resolution of signals from long-chain branching in polyethylene by 13C NMR at 188.6 MHz,” Macromolecules 32, 3817-3819 (1999).

Liu, Y. M., M. T. Shaw, and W. H. Tuminello, "Optimized data collection for determination of the MWD from the viscosity data of polymer melts," Polym. Eng. Sci. 38, 169-176 (1998).

Lohse, D. J., S. T. Milner, L. J. Fetters, M. Xenidou, N. Hadjichristidis, R. A. Mendelson, C. A. Garcia-Franco, and M. K. Lyon, "Well-defined, model long chain branched polyethylene. 2. Melt rheological behavior," Macromolecules 35, 3066-3075 (2002).

Malkin, A. Y., and A. E. Teishev, "Flow curve molecular-weight distribution-Is the solution of the inverse problem possible,” Polym. Eng. Sci. 31, 1590-1596 (1991).

Malmberg, A., C. Gabriel, T. Steffl, H. Munstedt, and B. Lofgren, "Long-chain branching in metallocenecatalyzed polyethylenes investigated by low oscillatory shear and uniaxial extensional rheometry," Macromolecules 35, 1038-1048 (2002).

McKee, M. G., S. Unal, G. L. Wilkes, and T. E. Long, "Branched polyesters: Recent advances in synthesis and performance," Prog. Polym. Sci. 30, 507-539 (2005).

Mead, D. W., "Determination of molecular-weight distributions of linear flexible polymers from linear viscoelastic material functions,” J. Rheol. 38, 1797-1827 (1994). 
Meng, Q. K., M. C. Heuzey, and P. J. Carreau, "Control of thermal degradation of polylactide/clay nanocomposites during melt processing by chain extension reaction," Polym. Degrad. Stab. 97, 2010-2020 (2012).

Mihai, M., M. A. Huneault, and B. D. Favis, "Rheology and extrusion foaming of chain-branched poly(lactic acid)," Polym. Eng. Sci. 50, 629-642 (2010).

Munari, A., G. Pezzin, and F. Pilati, "Linear and branched poly(butyleneisophthalate)—Activation-energy for melt flow," Rheol. Acta 29, 469-474 (1990).

Munstedt, H., "Dependence of the elongational behavior of polystyrene melts on molecular-weight and molecular-weight distribution," J. Rheol. 24, 847-867 (1980).

Munstedt, H., and D. Auhl, "Rheological measuring techniques and their relevance for the molecular characterization of polymers," J. Non-Newtonian Fluid Mech. 128, 62-69 (2005).

Najafi, N., M. C. Heuzey, P. J. Carreau, and P. M. Wood-Adams, "Control of thermal degradation of polylactide (PLA)-clay nanocomposites using chain extenders," Polym. Degrad. Stab. 97, 554-565 (2012).

Nobile, M. R., and F. Cocchini, "Evaluation of molecular weight distribution from dynamic moduli," Rheol. Acta 40, 111-119 (2001).

Piel, C., F. J. Stadler, J. Kaschta, S. Rulhoff, H. Munstedt, and W. Kaminsky, "Structure-property relationships of linear and long-chain branched metallocene high-density polyethylenes and SEC-MALLS," Macromol. Chem. Phys. 207, 26-38 (2006).

PLA Polymer 2002D, Polymer Technical Data Sheets (NatureWorks LLC, Minnetonka, MN, 2005), electronic file accessible at http://www.natureworksllc.com/Technical-Resources/2-Series

PLA Polymer 4032D, Polymer Technical Data Sheets (NatureWorks LLC, Minnetonka, MN, 2005), electronic file accessible at http://www.natureworksllc.com/Technical-Resources/4-Series

Raquez, J. M., R. Narayan, and P. Dubois, "Recent advances in reactive extrusion processing of biodegradable polymer-based compositions," Macromol. Mater. Eng. 293, 447-470 (2008).

Shaw, M. T., and W. H. Tuminello, "A closer look at the mwd-viscosity transform," Polym. Eng. Sci. 34, 159-165 (1994).

Shroff, R. N., and H. Mavridis, "Long-chain-branching index for essentially linear polyethylenes," Macromolecules 32, 8454-8464 (1999).

Stadler, F. J., and H. Munstedt, "Numerical description of shear viscosity functions of long-chain branched metallocene-catalyzed polyethylenes," J. Non-Newtonian Fluid Mech. 153, 203-203 (2008a).

Stadler, F. J., and H. Munstedt, "Terminal viscous and elastic properties of linear ethene/alpha-olefin copolymers,” J. Rheol. 52, 697-712 (2008b).

Stadler, F. J., C. Piel, J. Kaschta, S. Rulhoff, W. Kaminsky, and H. Munstedt, "Dependence of the zero shearrate viscosity and the viscosity function of linear high-density polyethylenes on the mass-average molar mass and polydispersity,” Rheol. Acta 45, 755-764 (2006).

Stadler, F. J., C. Piel, W. Kaminsky, and H. Munstedt, "Rheological characterization of long-chain branched polyethylenes and comparison with classical analytical methods," Macromol. Symp. 236, 209-218 (2006).

Sungsanit, K., N. Kao, S. N. Bhattacharya, and S. Pivsaart, "Physical and rheological properties of plasticized linear and branched PLA,” Korea-Aust. Rheol. J. 22, 187-195 (2010).

Tchir, W. J., and P. C. Saucier, "Accuracy in linear viscoelastic property measurement-Effects of instrument compliance and inertia revisited," in Antec 94-Plastics: Gate Way to the Future (CRC, SPE/ANTEC 1994 Proceedings, 1994), Vols 1-3, pp. 1205-1211.

Trinkle, S., and C. Friedrich, "Van Gurp-Palmen-plot: A way to characterize polydispersity of linear polymers," Rheol. Acta 40, 322-328 (2001).

Trinkle, S., P. Walter, and C. Friedrich, "Van Gurp-Palmen plot II-Classification of long chain branched polymers by their topology," Rheol. Acta 41, 103-113 (2002).

Tuminello, W. H., "Molecular-weight and molecular-weight distribution from dynamic measurements of polymer melts,” Polym. Eng. Sci. 26, 1339-1347 (1986).

Tuminello, W. H., and N. Cudremauroux, "Determining molecular-weight distributions from viscosity versus shear rate flow curves," Polym. Eng. Sci. 31, 1496-1507 (1991).

Van Gurp, M., and J. Palmen, "Time-temperature superposition for polymeric blends," in Proceedings of XIIth International Congress on Rheology, 1996, pp. 134-135. 
van Ruymbeke, E., V. Stephenne, D. Daoust, R. Godard, R. Keunings, and C. Bailly, "A sensitive method to detect very low levels of long chain branching from the molar mass distribution and linear viscoelastic response," J. Rheol. 49, 1503-1520 (2005).

Vega, J., M. Aguilar, J. Peón, D. Pastor, and J. Martínez-Salazar, "Effect of long chain branching on linearviscoelastic melt properties of polyolefins," e-Polymers 2, 624-658 (2002).

Vega, J. F., A. Santamaria, A. Munoz-Escalona, and P. Lafuente, "Small-amplitude oscillatory shear flow measurements as a tool to detect very low amounts of long chain branching in polyethylenes," Macromolecules 31, 3639-3647 (1998).

Wang, W. J., S. Kharchenko, K. Migler, and S. P. Zhu, "Triple-detector GPC characterization and processing behavior of long-chain-branched polyethylene prepared by solution polymerization with constrained geometry catalyst," Polymer 45, 6495-6505 (2004).

Wasserman, S. H., "Calculating the molecular-weight distribution from linear viscoelastic response of polymer melts," J. Rheol. 39, 601-625 (1995).

Wasserman, S. H., and W. W. Graessley, "Prediction of linear viscoelastic response for entangled polyolefin melts from molecular weight distribution,” Polym. Eng. Sci. 36, 852-861 (1996).

Wood-Adams, P., and S. Costeux, "Thermorheological behavior of polyethylene: Effects of microstructure and long chain branching," Macromolecules 34, 6281-6290 (2001).

Wood-Adams, P. M., and J. M. Dealy, "Use of rheological measurements to estimate the molecular weight distribution of linear polyethylene," J. Rheol. 40, 761-778 (1996).

Wood-Adams, P. M., and J. M. Dealy, "Using rheological data to determine the branching level in metallocene polyethylenes," Macromolecules 33, 7481-7488 (2000).

Wood-Adams, P. M., J. M. Dealy, A. W. deGroot, and O. D. Redwine, "Effect of molecular structure on the linear viscoelastic behavior of polyethylene," Macromolecules 33, 7489-7499 (2000).

Wu, C., Handbook of Size Exclusion Chromatography and Related Techniques, 2nd ed. (Taylor \& Francis eLibrary, NY, 2003).

Zhang, H., M. Tong, and R. Li, "Prediction of molecular weight scale and molecular weight distribution of cellulose using a rheology-based method," Polym. Eng. Sci. 48, 102-106 (2008).

Zimm, B. H., and W. H. Stockmayer, "The dimensions of chain molecules containing branches and rings," J. Chem. Phys. 17, 1301-1314 (1949). 